\title{
Diophantine approximations related to rational values of $G$-functions
}

\author{
by \\ Makoto NaGATa (Kyoto)
}

1. Introduction. A $G$-function, introduced by Siegel, is a solution of a linear differential equation satisfying some conditions. For example, algebraic functions over a number field and the Gauss hypergeometric series with rational parameters are $G$-functions. The detailed definition is given below.

In this paper, we will consider Diophantine properties related to rational values of $G$-functions.

Some irrationality results and some irrational measures for values of $G$ functions are known $([\mathrm{G}],[\mathrm{C}]$ et al.). First of all, we recall the results on special values of $G$-functions. We may say, in brief, that the results are like this:

Let $f(x)$ be a $G$-function and let $\varepsilon$ be any small positive real. Let $p, q \in$ $\mathbb{Z} \backslash\{0\}$ with $q$ large. If $\log |p|<O(\varepsilon \log |q|)$, then under some assumptions, the value $f(p / q)$ is irrational.

See $[\mathrm{G}],[\mathrm{C}]$ for more details.

Unfortunately, the condition "log $|p|<O(\varepsilon \log |q|)$ " seems artificial but indispensable. This is simply a technical reason: handling $G$-functions like $E$-functions. (See [Sh] for $E$-functions.)

Now we consider a question: "Find alternative natural conditions for statements relating to values of $G$-functions."

In this paper, we abandon regarding $G$-functions as an analogy of $E$ functions. From the viewpoint of radii of convergence, $G$-functions seem to be near algebraic functions, not near $E$-functions. Viewing $G$-functions as something like near-algebraic functions, we will introduce naively a set of their "rational points". We will then consider some Diophantine approximations on this set as properties related to rational values of $G$-functions. By

2000 Mathematics Subject Classification: Primary 11J25; Secondary 11D45.

Key words and phrases: $G$-functions. 
virtue of this approach, we will obtain some Diophantine properties under natural conditions.

Since algebraic functions defined over a number field are $G$-functions, the results obtained are extensions of Diophantine properties of rational points on algebraic curves.

Throughout this paper, $K$ denotes a number field with finite degree $[K: \mathbb{Q}]<\infty$.

1.1. Some results of the algebraic cases. In order to compare the algebraic cases and ours, we recall Liouville's inequality about rational points on algebraic curves and an estimate on the number of rational points on them. First, we recall the so-called Liouville inequality. To simplify, we consider only special cases with genus 0 .

Proposition 1 (Liouville's inequality). Let $g(y) \in K(y), n:=\operatorname{deg}_{y} g(y)$, $f(x, y):=x-g(y) \in K(x, y)$. Put

$$
\begin{aligned}
S_{1} & :=\{g(y) \in K \mid y \in K\} \\
& =\{x \in K \mid \text { there exists } y \in K \text { such that } f(x, y)=0\} .
\end{aligned}
$$

Fix $t \in K$ with $(d / d y) g(t) \neq 0$. Put $a:=g(t)$. Then there exists a positive constant $c>0$ such that

$$
|\alpha-a|>\frac{c}{H(\alpha)^{[K: \mathbb{Q}] / n}}
$$

for all $\alpha \in S_{1}$ with $\alpha \neq a$. Here the symbol $|\ldots|$ means the usual absolute valuation, and $H(\alpha)$ is the absolute Height of $\alpha$. We note that $c$ is independent of $\alpha$.

Proposition 1 is a slight extension of the original Liouville inequality. It is easy to verify it by using some properties of the height function (see, for example, [Se, 2.6]). One of the assertions of Proposition 1 is that this Diophantine approximation for rational points on an algebraic curve depends on the degree of the curve.

We remark that some sharper bounds for positive genus cases are known which use the Weil height, like Roth's theorem (see, for example, [Se, 7.3]).

In this paper, we will consider only the Liouville-type bound and its variants.

Some of our main results in $\S 1.3$ below (Theorem A and Corollary C) are extended versions of Proposition 1 for $G$-functions.

Next, we also recall an estimate on the number of rational points on algebraic curves.

Proposition 2 (estimate on the number of rational points on algebraic curves). Let $f(x, y) \in \mathbb{Q}[x, y]$ be an absolutely irreducible polynomial, and 
let $n:=\operatorname{deg}_{x} f(x, y)$. Put

$$
S_{2}:=\{x \in \mathbb{Q} \mid \text { there exists } y \in \mathbb{Q} \text { such that } f(x, y)=0\} .
$$

Then for any closed interval $[a, b] \subset \mathbb{R}$ which does not contain singular points of $S_{2}$, we have:

$$
\varlimsup_{B \rightarrow \infty} \frac{\log \#\left\{\zeta \in S_{2} \mid H(\zeta) \leq B, \zeta \in[a, b]\right\}}{\log B} \leq \frac{2}{n} .
$$

The upper limit in Proposition 2 has a trivial upper bound 2 by Schanuel's estimate (see [Se, 2.5]).

We remark that, due to Néron, Mumford and Faltings, it is well known that the upper bound is 0 if the curve defined by $f(x, y)=0$ has a positive genus.

The rest of our main results are Theorem B and Corollary D in $\S 1.3$, which are weaker but extended versions of Proposition 2 for $G$-functions.

1.2. G-functions and $G$-operators. Before stating our results, we recall the notions of $G$-function and $G$-operator. A $G$-function is a local solution of a linear differential equation, and a $G$-operator is the linear differential equation itself.

First, we recall the definition of $G$-functions.

Definition. A G-function is

(1) a power series solution $\in K[[x]]$ of a linear differential equation defined over $K(x)$ such that

(2) the Height of the tuples of 0 th, 1 st, .., ith coefficients of the power series grows at most geometrically in $i \in \mathbb{N}$.

It is known that algebraic functions defined over $K$ (which have a power series expression), polylogarithms, and the Gauss hypergeometric series with rational parameters are $G$-functions. Since algebraic functions are $G$-functions, the general properties of $G$-functions are also the properties of algebraic curves defined over a number field. For more information on $G$-functions, see e.g. [A], [B], [C].

Next, we recall the definition of $G$-operators. In brief, a $G$-operator is a linear differential equation satisfying an arithmetic condition. We consider the linear differential equation

$$
\frac{d}{d x} m=A m
$$

where $A \in M_{n}(K(x))$, a matrix of rational functions. In this paper, we always assume that $m$ is a column vector solution. 
Definition. We say $d / d x-A$ (or simply (EQ)) is a $G$-operator if

$$
\varlimsup_{m \rightarrow \infty} \sum_{v \nmid \infty} \frac{1}{m} \max _{i \leq m} \log ^{+}\left|\frac{1}{i !}{ }^{\mathrm{t}}\left(\frac{d}{d x}+{ }^{\mathrm{t}} A\right)^{i} I\right|_{v}<\infty,
$$

where $I$ is the identity matrix, $v$ in $\sum_{v \nmid \infty}$ runs over all non-Archimedean normalized (in the sense of $\S 2.1$ below) valuations of $K$. Here

$$
{ }^{\mathrm{t}}\left(\frac{d}{d x}+{ }^{\mathrm{t}} A\right){ }^{\mathrm{t}} B:=\left(\frac{d}{d x} B+B A\right) \quad \text { for } B \in M_{n}(K(x)),
$$

and $|\ldots|_{v}$ is the so-called Gauss norm.

For more information on $G$-operators, see e.g. [A], [C], [N1].

It might seem that the definition of $G$-operators depends on the choice of coordinates, but see [N3].

We note that $G$-functions are defined as power series; on the other hand, $G$-operators are defined by $A$ in (EQ), which is a matrix of rational functions. In other words, $G$-functions are local objects and $G$-operators are global objects.

Here we recall a fundamental fact: the notions of $G$-functions and of $G$ operators are equivalent under some conditions. In particular, under some natural conditions, if $m$, which is a local solution of (EQ), is a vector of $G$-functions, then (EQ) is a $G$-operator. We will use this fact in $\S 4.3$ below. See $[\mathrm{C}],[\mathrm{A}],[\mathrm{N} 1]$ for details.

1.3. Results. Now, we state our results. We will give their proofs in $\S 4$.

Let $d(x) \in \mathbb{Z}[x]$, a polynomial over the rational integers, be a common denominator of the components of $A$ in (EQ), that is, $d(x) A \in M_{n}\left(\mathcal{O}_{K}[x]\right)$ where $\mathcal{O}_{K}$ is the ring of integers of $K$.

We say that a function $f$ is analytic on a closed disk $D \subset \mathbb{C}$ if there exists an open disk $U$ with $D \subset U$ such that $f$ is analytic on $U$.

Theorem A. Let $D$ be a closed disk $\subset \mathbb{C}$ centered at $\zeta_{0} \in K$ with radius $<1 / 2$. For a vector solution $m(x)={ }^{\mathrm{t}}\left(f_{1}(x), \ldots, f_{n}(x)\right)$ of (EQ), suppose the following:

(0) (EQ) is a $G$-operator with $n \geq 2$.

(1) $m(x)$ is analytic on $D$ and $f_{1}(x), \ldots, f_{n}(x)$ are linearly independent over $\mathbb{C}(x)$.

(2) There exist no solutions of $d(x)=0$ on $D$. Put

$S_{K}:=\left\{\zeta \in D \cap K \mid\right.$ there exists $\kappa_{\zeta} \in \mathbb{C}, \neq 0$ such that $\left.\kappa_{\zeta} m(\zeta) \in K^{n}\right\}$, 
where $\kappa_{\zeta}$ depends on $m(\zeta)$. If $\zeta_{0} \in S_{K}$, then for any small $\varepsilon>0$, there exists an effective constant $c<\infty$ such that

$$
\left|\zeta_{0}-\zeta\right| \geq \frac{1}{H(\zeta)^{[K: \mathbb{Q}](1 / n+\varepsilon)}}
$$

for any $\zeta \in S_{K}$ with $H(\zeta) \geq c$. Here $c$ depends on $H\left(\zeta_{0}\right), A, \varepsilon, D$ and is independent of $\zeta$.

TheOREm B. Under the assumptions of Theorem A, we have

$$
\varlimsup_{B \rightarrow \infty} \frac{\log \#\left\{\zeta \in S_{K} \mid H(\zeta) \leq B\right\}}{\log B} \leq \frac{4}{n}[K: \mathbb{Q}] .
$$

The trivial upper bound in Theorem B is $2[K: \mathbb{Q}]$ by Schanuel's estimate.

We recall $\S 1.1$. Although the meaning of $n$ is different, Theorem A corresponds to Proposition 1 and Theorem B to Proposition 2.

We also obtain non-algebraic cases as corollaries.

COROLlary C. Under the assumptions of Theorem A, assume moreover that:

(3) $f_{1}(x), \ldots, f_{n}(x)$ are homogeneously algebraically independent over $\mathbb{C}(x)$.

(4) $f_{1}(x), \ldots, f_{n}(x)$ are $G$-functions.

If $\zeta_{0} \in S_{K}$, then for any small $\varepsilon>0$, there exists an effective constant $c<\infty$ such that

$$
\left|\zeta_{0}-\zeta\right| \geq \frac{1}{H(\zeta)^{\varepsilon}}
$$

for any $\zeta \in S_{K}$ with $H(\zeta) \geq c$. Here $c$ depends on $H\left(\zeta_{0}\right), A, \varepsilon, D$ and is independent of $\zeta$.

Corollary D. Under the assumptions of Corollary $\mathrm{C}$, we have

$$
\varlimsup_{B \rightarrow \infty} \frac{\log \#\left\{\zeta \in S_{K} \mid H(\zeta) \leq B\right\}}{\log B}=0 .
$$

We remark that the estimates in Corollaries $\mathrm{C}$ and $\mathrm{D}$ (non-algebraic function cases) are similar to the cases of algebraic curves with positive genus (non-rational function cases). See $\S 1.1$ again.

1.4. Examples. Here we show some examples. Example 1 concerns algebraic functions, and Example 2 deals with non-algebraic functions.

EXAMPLE 1 (Fermat's curves). Let $k$ be a natural number $\geq 2$. We consider the curve $x^{k}+y^{k}=1$. Thus, $y=\sqrt[k]{1-x^{k}}$ is not a rational function. Moreover,

$$
\frac{d}{d x}\left(\begin{array}{l}
1 \\
y
\end{array}\right)=\left(\begin{array}{cc}
0 & 0 \\
0 & \frac{x^{k-1}}{x^{k}-1}
\end{array}\right)\left(\begin{array}{l}
1 \\
y
\end{array}\right) .
$$


Now let $n$ be 2 , let $\zeta_{0}$ be in $K$ with $\left|\zeta_{0}\right|<1 / 2$ such that $\sqrt[k]{1-\zeta^{k}} \in K$ (e.g., $\zeta_{0}=0$ ), and define $D:=\left\{z \in \mathbb{C}|| z-\zeta_{0} \mid \leq 1 / 3\right\}$. The set $S_{K}$ in Theorem A is

$$
S_{K}:=\left\{\zeta \in D \cap K \mid y=\sqrt[k]{1-\zeta^{k}} \in K\right\}
$$

Therefore for any small $\varepsilon>0$ there exists an effective constant $c<\infty$ such that

$$
\left|\zeta_{0}-\zeta\right| \geq \frac{1}{H(\zeta)^{[K: \mathbb{Q}](1 / 2+\varepsilon)}} \quad \text { for any } \zeta \in S_{K} \text { with } H(\zeta) \geq c .
$$

We remark that the effectiveness of $c$ in Example 1 should be distinguished from the ineffective finiteness results on the number of rational points on some curves.

Next, we show examples concerning the Gauss hypergeometric series.

We consider the Gauss hypergeometric series with parameters $\alpha, \beta, \gamma \in \mathbb{Q}$ $(\gamma \neq-1,-2, \ldots)$,

$$
F(\alpha, \beta, \gamma ; x):=\sum_{i=1}^{\infty} \frac{(\alpha)_{i}(\beta)_{i}}{(\gamma)_{i} i !} x^{n} .
$$

Let $F$ denote $F(\alpha, \beta, \gamma ; x)$ for simplicity. The function $F$ satisfies the linear differential equation

$$
x(1-x) y^{\prime \prime}=((1+\alpha+\beta) x-\gamma) y^{\prime}+\alpha \beta y .
$$

ExAmple 2 (rational values of the logarithmic derivative of the Gauss hypergeometric series). Let $D$ be a closed disk with radius $<1 / 2$ which is contained in the open disk centered at the origin with radius 1 . Assume that $D$ does not contain the origin. Set

$$
S_{K}:=\left\{x \in D \cap K \mid F(x) \neq 0, F^{\prime}(x) / F(x) \in K\right\} .
$$

Assume that there exists a solution of (hyp) which is not an algebraic function (this is obviously the case if $F$ is not algebraic), and assume that $\alpha, \beta, \gamma-\alpha, \gamma-\beta \notin \mathbb{Z}$. Then:

(a) If $\zeta_{0} \in S_{K}$, then for any small $\varepsilon>0$, there exists $c<\infty$, effective, such that

$$
\begin{aligned}
\left|\zeta_{0}-\zeta\right|> & \frac{1}{H(\zeta)^{\varepsilon}} \quad \text { for any } \zeta \in S_{K} \text { with } H(\zeta)>c . \\
& \varlimsup_{B \rightarrow \infty} \frac{\log \#\left\{\zeta \in S_{K} \mid H(\zeta) \leq B\right\}}{\log B}=0 .
\end{aligned}
$$

(For transcendental properties of values of the Gauss hypergeometric series itself, see $[\mathrm{Wo}]$.)

Proof of Example 2. The radius of convergence of $F$ at the origin is 1 . Since $F$ satisfies (hyp), the vector $m=\left(F, F^{\prime}\right)$ is a solution of (EQ) with 
$n=2, A \in M_{2}(\mathbb{Q}(x)), d(x)=x(x-1)$. Moreover, by Theorem 5 in [BMV], $F$ and $F^{\prime}$ are algebraically independent over $\mathbb{C}(x)$. Since the set $S_{K}$ of Example 2 corresponds to $S_{K}$ of Corollaries $\mathrm{C}$ and D, we obtain the assertion of Example 2.

For the readers interested in periodic functions, we add an example of a special case: elliptic integrals of the first and second kind. Let

$$
y(x):=\frac{1}{\pi} \int_{0}^{1} \frac{d t}{\sqrt{t(1-t)(1-t x)}}, \quad w(x):=\frac{1}{\pi} \int_{0}^{1} \frac{1-t x}{\sqrt{t(1-t)(1-t x)}} d t
$$

and let $D$ be as in Example 2. Set

$$
S_{K}:=\{\zeta \in D \cap K \mid w(x) \neq 0, y(\zeta) / w(\zeta) \in K\} .
$$

Then the estimates (a) and (b) of Example 2 hold for this $S_{K}$.

It is easy to verify this special case. We consider the case of $\alpha=-1 / 2$, $\beta=1 / 2, \gamma=1$ in Example 2. Then the second assumption in Example 2 holds. The first assumption in Example 2 also holds, since there exists a solution of (hyp) with a logarithmic singularity at the origin. Moreover,

$y(x)=F(1 / 2,1 / 2,1 ; x), \quad w(x)=F(-1 / 2,1 / 2,1 ; x), \quad x w^{\prime}=-\frac{1}{2}(w-y)$.

Therefore for $\zeta \in K$ with $0<|\zeta|<1$, the conditions $w^{\prime}(\zeta) / w(\zeta) \in K$ and $y(\zeta) / w(\zeta) \in K$ are equivalent, that is, the $S_{K}$ in Example 2 and here are the same. This completes the proof of this case.

The content of this paper is the following: In $\S 2$, we will show some properties of $G$-operators, and recall some known results which will be used in $\S 3$ and $\S 4$. Next in $\S 3$, we will give a fundamental inequality (Lemma 3.4). This section requires long calculations, but the inequality makes our proofs simple.

In $\S 4$, we will give the proofs of Theorems A, B, and Corollaries C and D. We will also show Liouville's inequality for $G$-functions on moving targets (Theorem E).

2. Preliminaries. In this section, we recall the height functions, show some properties of $G$-operators, and state some related results which are necessary for the later sections.

2.1. Heights. We use the symbol $|\ldots|$ for the usual absolute valuation: $|s+\sqrt{-1} t|:=\sqrt{s^{2}+t^{2}}, s, t \in \mathbb{R}$. Let $K$ be a number field with finite degree, and set $d_{K}:=[K: \mathbb{Q}]$. If $v$ is a place of $K$, the symbol $|\ldots|_{v}$ denotes the valuation at $v$, and $K_{v}$ the completion of $K$ at $v$.

We use the notation $v \mid p$ if $v$ is an extension of a prime number $p$ which is a non-Archimedean place on $\mathbb{Q}$. We also use the notations $v \mid \infty$ if $v$ is an Archimedean place, and $v \nmid \infty$ if $v$ is a non-Archimedean place. 
We assume that $|\ldots|_{v}$ is normalized as follows: If $v \mid p$, then we put $d_{v}:=\left[K_{v}: \mathbb{Q}_{p}\right]$ and define $|p|_{v}:=p^{-d_{v} / d_{K}}$. If $v \mid \infty$, then for $\alpha \in K$ and for a $\mathbb{Q}$-homomorphism $\sigma: K \rightarrow \mathbb{C}$ such that $|\sigma(\alpha)|$ and $|\ldots|_{v}$ induce the same topology on $K$, we put

$$
d_{v}:= \begin{cases}1 & \text { if } \sigma(K) \subset \mathbb{R}, \\ 2 & \text { if } \sigma(K) \not \subset \mathbb{R},\end{cases}
$$

and we define $|\alpha|_{v}:=|\sigma(\alpha)|^{d_{v} / d}$. Here $|\ldots|$ is the usual valuation.

In the latter case, we will use the symbol $|\ldots|_{\sigma}$ (resp. $d_{\sigma}$ ) instead of $|\ldots|_{v}\left(\right.$ resp. $\left.d_{v}\right):|\ldots|_{\sigma}:=|\sigma(\ldots)|^{d_{v} / d}=|\sigma(\ldots)|^{d_{\sigma} / d}$. In particular, in the case of $\sigma=$ id (the identity homomorphism), we write $|\ldots|_{1}$ (resp. $d_{1}$ ) instead of $|\ldots|_{\text {id }}:|\ldots|_{1}=|\ldots|_{\text {id }}:=|\ldots|^{d_{\text {id }} / d}=|\ldots|^{d_{1} / d}$.

The following is obvious: for $\alpha \in K$, if $|\alpha|<1$, then $|\alpha|_{1}=|\alpha|^{d_{1} / d_{K}}$ $\leq|\alpha|^{1 / d_{K}}$.

From now on, let $M_{K}$ be the set of all normalized valuations of $K$, and set $M_{K}^{0}=\left\{v \in M_{K} \mid v \nmid \infty\right\}, M_{K}^{\infty}=\left\{v \in M_{K}|v| \infty\right\}, M_{K}^{1}=\left\{v \in M_{K} \mid\right.$ $v \mid \infty, v \neq \mathrm{id}\}$.

The following is the product formula on a number field: if $\alpha \in K \backslash\{0\}$, then $\prod_{v \in M_{K}}|\alpha|_{v}=1$.

Next, for a non-negative integer $n$ and $\bar{\alpha}:=\left(\alpha_{0}, \alpha_{1}, \ldots, \alpha_{n}\right) \in K^{n+1} \backslash\{\overline{0}\}$, we define

$$
\begin{aligned}
H(\bar{\alpha}) & :=\prod_{v \in M_{K}} \max \left(\left|\alpha_{0}\right|_{v}, \ldots,\left|\alpha_{n}\right|_{v}\right), \\
h(\bar{\alpha}) & :=\sum_{v \in M_{K}} \log \max \left(\left|\alpha_{0}\right|_{v}, \ldots,\left|\alpha_{n}\right|_{v}\right) .
\end{aligned}
$$

By the product formula, $h((x, y))=h((1, y / x))$ if $x, y \in K$ and $x \neq 0$.

For $\alpha \in K$, we call $H(\alpha):=H((1, \alpha))=\prod_{v} \max \left(1,|\alpha|_{v}\right)$ the Height of $\alpha$, and $h(\alpha):=h((1, \alpha))=\sum_{v} \log \max \left(1,|\alpha|_{v}\right)$ the (logarithmic) height of $\alpha$. Thus $h(\alpha)=h(1 / \alpha)$ if $\alpha \in K \backslash\{0\}$. We note that $h(\alpha)$ is different from $h((\alpha))(h((\alpha))=0$ if $\alpha \neq 0)$.

We use the notation $\log ^{+} a:=\log \max (1, a)$ if $a \in \mathbb{R}$. Thus $h(\alpha)=$ $\sum_{v \in M_{K}} \log ^{+}|\alpha|_{v}$.

For a polynomial $P:=\alpha_{0}+\alpha_{1} x+\ldots+\alpha_{n} x^{n} \in K[x]$, we put

$$
\begin{aligned}
H(P) & :=\prod_{v \in M_{K}} \max \left(\left|\alpha_{0}\right|_{v}, \ldots,\left|\alpha_{n}\right|_{v}\right), \\
h(P) & :=\sum_{v \in M_{K}} \log \max \left(\left|\alpha_{0}\right|_{v}, \ldots,\left|\alpha_{n}\right|_{v}\right) .
\end{aligned}
$$

Let $M_{s, t}(K[x])$ be the set of $s \times t$-matrices whose components are in $K[x]$. 
For $\phi:=A_{0}+A_{1} x+\ldots+A_{n} x^{n} \in M_{s, t}(K[x]),\left(a_{i, j, k}\right):=A_{k} \in M_{s, t}(K)$ for $k=0,1, \ldots, n, a_{i, j, k} \in K$, we put

$$
H(\phi):=\prod_{v \in M_{K}} \max _{i, j, k}\left(\left|a_{i, j, k}\right|_{v}\right), \quad h(\phi):=\sum_{v \in M_{K}} \log \max _{i, j, k}\left(\left|a_{i, j, k}\right|_{v}\right) .
$$

We note here that in the definition of the height of polynomials $\log ^{+}$is not used, and of course $v$ in the summation runs also over $v \mid \infty$.

2.2. G-operators revisited. We denote by $K(x)$ the rational function field in one variable over $K$. For $n \in \mathbb{N}$, and for an $n \times n$-matrix $A \in M_{n}(K(x))$ of rational functions, we consider the linear differential equation

$$
\frac{d}{d x} m=A m \text {. }
$$

Here, we assume that $m$ is a column vector solution.

Moreover we denote by $\mathcal{O}_{K}$ the integer ring of $K$, and we fix a polynomial $d$ as a denominator of $A$ :

$$
d=d(x) \in \mathbb{Z}[x] \quad \text { such that } d A \in M_{n}\left(\mathcal{O}_{K}[x]\right) .
$$

Let $\gamma_{1}$ be the geometric (logarithmic) height of $A$ in (EQ),

$$
\gamma_{1}:=\max (\operatorname{deg} d, \operatorname{deg} d A) .
$$

Here $\operatorname{deg} d A$ means the maximal degree of its components.

We note that $d$ is just one of the denominators of $A$. In this paper, it is not necessary that $\gamma_{1}$ be minimal. This remark will be used in the proof of Corollaries C and D.

Now we go back to valuations. For $P=a_{0}+a_{1} x+\ldots+a_{n} x^{n} \in K[x]$, we put

$$
|P|_{v}:=\max \left(\left|a_{0}\right|_{v}, \ldots,\left|a_{n}\right|_{v}\right)
$$

for $v \in M_{K}$. Thus $h(P)=\sum_{v \in M_{K}} \log |P|_{v}$.

For $v \nmid \infty$, it is known that $|P Q|_{v}=|P|_{v}|Q|_{v}$ for $P, Q \in K[x]$. This fact is the so-called Gauss lemma ([L1, p. 55, Proposition 2.1]).

Let $I$ be the identity matrix.

If we defined a geometric $G$-operator to be such that the degrees (as the logarithmic geometric height) of $J_{\mu}$ in Lemma 2.1 below grow at most arithmetically, the lemma would state that every (EQ) is a geometric $G$ operator. (Therefore this definition is redundant.)

Lemma 2.1. Let $A$ be a matrix in (EQ), and let d be a common denominator of $A$ as in (2.1). For $\mu=0,1, \ldots$, put

$$
J_{\mu}:=\left(\frac{d}{d x}+{ }^{\mathrm{t}} A\right)^{\mu} I
$$


where $J_{0}=I,\left(d / d x+{ }^{\mathrm{t}} A\right) J_{\mu}:=(d / d x) J_{\mu}+{ }^{\mathrm{t}} A J_{\mu}$, that is, $J_{1}={ }^{\mathrm{t}} A, J_{2}=$ $(d / d x){ }^{\mathrm{t}} A+{ }^{\mathrm{t}} A{ }^{\mathrm{t}} A, \ldots$ Then for $\mu=0,1, \ldots$ we have

$$
\begin{gathered}
d^{\mu+1} J_{\mu+1}=-\mu\left(\frac{d}{d x} d\right)\left(d^{\mu} J_{\mu}\right)+d\left(\frac{d}{d x}+{ }^{\mathrm{t}} A\right)\left(d^{\mu} J_{\mu}\right), \\
d^{\mu} J_{\mu} \in M_{n}\left(\mathcal{O}_{K}[x]\right), \quad \operatorname{deg}\left(d^{\mu} J_{\mu}\right) \leq \mu \gamma_{1} .
\end{gathered}
$$

Proof. Since $d$ is in the center of $M_{n}(K(x))$, we have

$$
\begin{aligned}
\left(\frac{d}{d x}+{ }^{\mathrm{t}} A\right)\left(d^{\mu} J_{\mu}\right) & =\left(\frac{d}{d x} d^{\mu}\right) J_{\mu}+d^{\mu} \frac{d}{d x} J_{\mu}+{ }^{\mathrm{t}} A d^{\mu} J_{\mu} \\
& =\mu\left(\frac{d}{d x} d\right) d^{\mu-1} J_{\mu}+d^{\mu}\left(\frac{d}{d x}+{ }^{\mathrm{t}} A\right) J_{\mu} .
\end{aligned}
$$

By multiplying the last relations by $d$, we obtain (2.3).

Next, for $\mu=0,(2.4)$ holds as $J_{0}=I$. For $\mu=1$, from

$$
d J_{1}=d\left(\frac{d}{d x}+{ }^{\mathrm{t}} A\right) I=d^{\mathrm{t}} A
$$

and by (2.1), (2.2), both $d J_{1} \in M_{n}\left(\mathcal{O}_{K}[x]\right)$ and $\operatorname{deg}\left(d J_{1}\right) \leq \gamma_{1}$ hold.

We continue by induction on $\mu$. We assume that (2.4) holds for $\mu \geq 1$. Since $d \in \mathbb{Z}[x]$ and $d A \in M_{n}\left(\mathcal{O}_{K}[x]\right)$, we obtain $d^{\mu+1} J_{\mu+1} \in M_{n}\left(\mathcal{O}_{K}[x]\right)$ by (2.3) from the assumption $d^{\mu} J_{\mu} \in M_{n}\left(\mathcal{O}_{K}[x]\right)$. Moreover, by (2.3), $\operatorname{deg}\left(d^{\mu+1} J_{\mu+1}\right)$

$\leq \max \left(\max \left(\gamma_{1}-1,0\right)+\operatorname{deg}\left(d^{\mu} J_{\mu}\right), \gamma_{1}+\max \left(\operatorname{deg}\left(d^{\mu} J_{\mu}\right)-1,0\right), \gamma_{1}+\operatorname{deg}\left(d^{\mu} J_{\mu}\right)\right)$. From the assumption $\operatorname{deg}\left(d^{\mu} J_{\mu}\right) \leq \mu \gamma_{1}$, we obtain $\operatorname{deg}\left(d^{\mu+1} J_{\mu+1}\right) \leq$ $(\mu+1) \gamma_{1}$.

By Lemma 2.1, we have $\left(d^{\mu} / \mu !\right)\left(d / d x+{ }^{\mathrm{t}} A\right)^{\mu} I \in M_{n}(K[x])$, a matrix of polynomials, and thus for $m \in \mathbb{N} \cup\{0\}$, we can define the real-valued functions $G_{0}, G_{\infty}, G_{1}$ by

$$
\begin{aligned}
G_{0}(m) & :=\prod_{v \nmid \infty} \max _{\mu=0,1, \ldots, m}\left|\frac{d^{\mu}}{\mu !}\left(\frac{d}{d x}+{ }^{\mathrm{t}} A\right)^{\mu} I\right|_{v}, \\
G_{\infty}(m) & :=\prod_{v \mid \infty} \max _{\mu=0,1, \ldots, m}\left|\frac{d^{\mu}}{\mu !}\left(\frac{d}{d x}+{ }^{\mathrm{t}} A\right)^{\mu} I\right|_{v}, \\
G_{1}(m) & :=\prod_{\substack{v \nmid \infty \\
v \neq 1}} \max _{\mu=0,1, \ldots, m}\left|\frac{d^{\mu}}{\mu !}\left(\frac{d}{d x}+{ }^{\mathrm{t}} A\right)^{\mu} I\right|_{v} .
\end{aligned}
$$

Remark. (0) Since the number of $v \in M_{K}$ with $\left|\left(d^{\mu} / \mu !\right)\left(d / d x+{ }^{\mathrm{t}} A\right)^{\mu} I\right|_{v}$ $\neq 1$ is finite, each product above is finite. 
(1) Since $\left(d^{\mu} / \mu !\right)\left(d / d x+{ }^{\mathrm{t}} A\right)^{\mu} I=I$ for $\mu=0$, for $v \in M_{K}$ we have

$$
\max _{\mu=0,1, \ldots, m}\left|\frac{d^{\mu}}{\mu !}\left(\frac{d}{d x}+{ }^{\mathrm{t}} A\right)^{\mu} I\right|_{v} \geq 1 .
$$

Therefore each $G_{0}(m), G_{\infty}(m), G_{1}(m)$ is an increasing function of $m$. In particular, $G_{0}(m) \geq 1, G_{\infty}(m) \geq 1, G_{1}(m) \geq 1$. Moreover it is obvious that

$$
G_{1}(m) \leq G_{1}(m) \max _{\mu=0,1, \ldots, m}\left|\frac{d^{\mu}}{\mu !}\left(\frac{d}{d x}+{ }^{\mathrm{t}} A\right)^{\mu} I\right|_{1}=G_{\infty}(m) .
$$

(2) Since $d \in \mathbb{Z}[x]$, we have $|d|_{v} \leq 1$ for $v \nmid \infty$. Hence by Gauss' lemma, for $\mu=0,1, \ldots$ we have

$$
\left|\frac{d^{\mu}}{\mu !}\left(\frac{d}{d x}+{ }^{\mathrm{t}} A\right)^{\mu} I\right|_{v} \leq\left|\frac{1}{\mu !}\left(\frac{d}{d x}+{ }^{\mathrm{t}} A\right)^{\mu} I\right|_{v} .
$$

Therefore

$$
G_{0}(m) \leq \prod_{v \nmid \infty} \max _{\mu=0,1, \ldots, m}\left|\frac{1}{\mu !}\left(\frac{d}{d x}+{ }^{\mathrm{t}} A\right)^{\mu} I\right|_{v},
$$

that is, if (EQ) is a $G$-operator, then there exists a finite constant $c<\infty$, independent of $m$, such that $G_{0}(m) \leq C_{0}^{m}$ for $m=0,1, \ldots$

Again we could define an Archimedean $G$-operator to have $G_{\infty}(m)$ growing at most geometrically for $m=0,1, \ldots$ The following Lemma 2.2 states that every (EQ) is an Archimedean $G$-operator. (Therefore this definition is also redundant.)

Lemma 2.2. There exists a constant $C<\infty$, independent of $m$, such that $G_{\infty}(m) \leq C^{m}$ for $m=0,1, \ldots$

Proof. From (2.1), (2.2), let $d_{j} \in \mathbb{Z}$ and $\alpha_{j} \in M_{n}\left(\mathcal{O}_{K}\right)$ be such that

$$
d=\sum_{j=0}^{\gamma_{1}} d_{j} x^{j}, \quad d^{\mathrm{t}} A=\sum_{j=0}^{\gamma_{1}} \alpha_{j} x^{j} .
$$

According to (2.4) in Lemma 2.1, we define $A_{j, \mu}$ in $M_{n}\left(\mathcal{O}_{K}\right)$ by

$$
d^{\mu} J_{\mu}=\sum_{j=0}^{\mu \gamma_{1}} A_{j, \mu} x^{j} .
$$

Since $(d / d x) d=\sum_{j=0}^{\gamma_{1}} j d_{j} x^{j-1}$, we have

$$
\left(\frac{d}{d x} d\right)\left(d^{\mu} J_{\mu}\right)=\sum_{j=0}^{\gamma_{1}} j d_{j} x^{j-1} \sum_{k=0}^{\mu \gamma_{1}} A_{k, \mu} x^{k}=\sum_{t=0}^{(\mu+1) \gamma_{1}} \sum_{\substack{j+k=t \\ 0 \leq j \leq \gamma_{1} \\ 0 \leq k \leq \mu \gamma_{1}}} j d_{j} A_{k, \mu} x^{t-1}
$$


Next, since $(d / d x)\left(d^{\mu} J_{\mu}\right)=\sum_{j=0}^{\mu \gamma_{1}} j A_{j, \mu} x^{j-1}$, we also have

$$
\begin{aligned}
d\left(\frac{d}{d x}\left(d^{\mu} J_{\mu}\right)\right) & =\sum_{j=0}^{\gamma_{1}} d_{j} x^{j} \sum_{k=0}^{\mu \gamma_{1}} k A_{k, \mu} x^{k-1} \\
& =\sum_{t=0}^{(\mu+1) \gamma_{1}} \sum_{\substack{j+k=t \\
0 \leq j \leq \gamma_{1} \\
0 \leq k \leq \mu \gamma_{1}}} k d_{j} A_{k, \mu} x^{t-1} .
\end{aligned}
$$

Finally, we have

$$
d^{\mathrm{t}} A\left(d^{\mu} J_{\mu}\right)=\sum_{j=0}^{\gamma_{1}} \alpha_{j} x^{j} \sum_{k=0}^{\mu \gamma_{1}} A_{k, \mu} x^{k}=\sum_{t=0}^{(\mu+1) \gamma_{1}} \sum_{\substack{j+k=t \\ 0 \leq j \leq \gamma_{1} \\ 0 \leq k \leq \mu \gamma_{1}}} \alpha_{j} A_{k, j} x^{t} .
$$

By (2.3) and (2.6)-(2.8), we obtain

$$
\begin{aligned}
d^{\mu+1} J_{\mu+1} & =-\mu\left(\frac{d}{d x} d\right) d^{\mu} J_{\mu}+d\left(\frac{d}{d x}+{ }^{\mathrm{t}} A\right)\left(d^{\mu} J_{\mu}\right) \\
& =\sum_{t=0}^{(\mu+1) \gamma_{1}} \sum_{\substack{j+k=t \\
0 \leq j \leq \gamma_{1} \\
0 \leq k \leq \mu \gamma_{1}}}-\mu j d_{j} A_{k, \mu} x^{t-1}+k d_{j} A_{k, \mu} x^{t-1}+\alpha_{j} A_{k, \mu} x^{t} .
\end{aligned}
$$

For $t=0,1, \ldots,(\mu+1) \gamma_{1}-1$, the coefficient of $x^{t}$ in the last equation is

$$
-\sum_{\substack{j+k=t+1 \\ 0 \leq j \leq \gamma_{1} \\ 0 \leq k \leq \mu \gamma_{1}}}\left(\mu j d_{j} A_{k, \mu}-k d_{j} A_{k, \mu}\right)+\sum_{\substack{j+k=t \\ 0 \leq j \leq \gamma_{1} \\ 0 \leq k \leq \mu \gamma_{1}}} \alpha_{j} A_{k, \mu} .
$$

For the remaining case: $t=(\mu+1) \gamma_{1}$, the coefficient of $x^{t}$ is

$$
\sum_{\substack{j+k=(\mu+1) \gamma_{1} \\ 0 \leq j \leq \gamma_{1} \\ 0 \leq k \leq \mu \gamma_{1}}} \alpha_{j} A_{k, \mu}=\alpha_{\gamma_{1}} A_{\mu \gamma_{1}, \mu} .
$$

Now, for $v \in M_{K}$, we define

$$
\begin{gathered}
d(v):=\max _{j=0, \ldots, \gamma_{1}}\left|d_{j}\right|_{v}, \quad \alpha(v):=\max _{j=0, \ldots, \gamma_{1}}\left|\alpha_{j}\right|_{v}, \\
A(\mu, v):=\max _{k=0, \ldots, \mu \gamma_{1}}\left|A_{k, \mu}\right|_{v} .
\end{gathered}
$$

We consider only the cases of $v \mid \infty$. As $\alpha_{j} \in M_{n}(K)$ and $v \mid \infty$, for $j=0, \ldots, \gamma_{1}, k=0, \ldots, \gamma_{1} \mu$, we have

$$
\begin{gathered}
\left|\mu j d_{j} A_{k, \mu}\right|_{v} \leq\left.\left.|\mu|_{v}\right|_{1}\right|_{v} d(v) A(\mu, v), \quad\left|k d_{j} A_{k, \mu}\right|_{v} \leq\left.\left.|\mu|_{v}\right|_{1}\right|_{v} d(v) A(\mu, v), \\
\left|\alpha_{j} A_{k, \mu}\right|_{v} \leq|n|_{v} \alpha(v) A(\mu, v) .
\end{gathered}
$$


For any $t$, the number of terms in

$$
\sum_{\substack{j+k=t+1 \\ 0 \leq j \leq \gamma_{1} \\ 0 \leq k \leq \mu \gamma_{1}}}
$$

is at most $\gamma_{1}+1$ because $0 \leq j \leq \gamma_{1}$.

Now we will estimate (2.9) and (2.10). The value of $|\ldots|_{v}$ at (2.9) satisfies

$$
|(2.9)|_{v} \leq\left|\gamma_{1}+1\right|_{v}|3|_{v} \max \left(|\mu|_{v}\left|\gamma_{1}\right|_{v} d(v),|n|_{v} \alpha(v)\right) A(\mu, v) .
$$

The value of $|\ldots|_{v}$ at $(2.10)$ satisfies

$$
|(2.10)|_{v} \leq\left|\gamma_{1}+1\right|_{v}|n|_{v} \alpha(v) A(\mu, v) .
$$

We note that these are estimates of the coefficients of $x^{t}$ in $d^{\mu+1} J_{\mu+1}$.

Now, let $A_{j, \mu+1} \in M_{n}\left(\mathcal{O}_{K}\right)$ be such that $d^{\mu+1} J_{\mu+1}=\sum_{j=0}^{(\mu+1) \gamma_{1}} A_{j, \mu+1} x^{j}$. With the above arguments, we obtain

$$
\begin{aligned}
\max _{k=0, \ldots,(\mu+1) \gamma_{1}}\left|A_{k, \mu+1}\right|_{v} & \leq \max \left(|(2.9)|_{v},|(2.10)|_{v}\right) \\
& \leq\left|\gamma_{1}+1\right|_{v}|3|_{v} \max \left(|\mu|_{v}\left|\gamma_{1}\right|_{v} d(v),|n|_{v} \alpha(v)\right) A(\mu, v) .
\end{aligned}
$$

Put

$$
\beta_{v}:=\left|\gamma_{1}+1\right|_{v} \max \left(\left|\gamma_{1}\right|_{v} d(v),|n|_{v} \alpha(v)\right)
$$

Then

$$
A(\mu+1, v)=\max _{k=0, \ldots,(\mu+1) \gamma_{1}}\left|A_{k, \mu+1}\right|_{v} \leq|3|_{v} \beta_{v}|\mu|_{v} A(\mu, v) .
$$

Therefore

$$
A(\mu, v) \leq\left(|3|_{v} \beta_{v}\right)^{\mu-1}|(\mu-1) !|_{v} A(1, v) .
$$

Since $d J_{1}=d^{\mathrm{t}} A=\sum_{j=0}^{\gamma_{1}} \alpha_{j} x^{j}$, we have $A(1, v) \leq \beta_{v}$.

The aim of this proof is to estimate

$$
\left|\frac{d^{\mu}}{\mu !}\left(\frac{d}{d x}+{ }^{\mathrm{t}} A\right)^{\mu} I\right|_{v}=\left|\frac{d^{\mu}}{\mu !} J_{\mu}\right|_{v} \leq \frac{1}{|\mu !|_{v}} A(\mu, v) .
$$

Since $|3|_{v} \geq 1$ for $v \mid \infty$ and since $|\mu|_{v} \geq 1$, we have an estimate

$$
\frac{1}{|\mu !|_{v}} A(\mu, v) \leq\left(|3|_{v} \beta_{v}\right)^{\mu} \frac{1}{|\mu|_{v}} \leq\left(|3|_{v} \beta_{v}\right)^{\mu} .
$$

Therefore

$$
G_{\infty}(m) \leq \prod_{v \mid \infty} \max _{\mu=0, \ldots, m}\left(1,\left(|3|_{v} \beta_{v}\right)^{\mu}\right)=\left(\prod_{v \mid \infty} \max \left(1,\left(|3|_{v} \beta_{v}\right)\right)\right)^{m} .
$$

This implies that there exists a finite constant $C$, depending only on $A$, but independent of $m$, such that $G_{\infty}(m) \leq C^{m}$. 
If (EQ) is a $G$-operator, Lemmas 2.1 and 2.2 assert that there exists a finite constant $C$ such that

$$
\exp \left(\operatorname{deg}\left(d^{m} J_{m}\right)\right) \leq C^{m}, \quad G_{\infty}(m) \leq C^{m}, \quad G_{0}(m) \leq C^{m}
$$

for $m=0,1, \ldots$

The fact that "a geometric property holds together with an arithmetic property" is one of the most important features of $G$-operators; here a geometric property means $\exp \left(\operatorname{deg}\left(d^{m} J_{m}\right)\right) \leq C^{m}$, and an arithmetic property means $G_{\infty}(m) \leq C^{m}$ and $G_{0}(m) \leq C^{m}$.

This fact will be worked out effectively in $\S 3$.

The next proposition is due to Chudnovsky-Chudnovsky. We will use it for our proofs of Corollaries C and D.

We note again that a $G$-function is defined as a local object (a power series solution); nevertheless Proposition 2.3 shows that it involves a $G$ operator which is defined as a global object.

Proposition $2.3([\mathrm{C}])$. Let $m=\left(f_{1}, \ldots, f_{n}\right)$ be a vector solution of (EQ). If all $f_{i}$ are $G$-functions, and if they are linearly independent over $\mathbb{C}(x)$, then (EQ) is a $G$-operator.

See $[\mathrm{C}]$ and $[\mathrm{A}]$ for the proof.

2.3. Some known results. We recall a variant of Liouville's inequality, which is stated in function-theoretic terms.

The following Proposition 2.4 is a special case of [O, Theorem IV(ii)], and it is an extended version of a special case of Shidlovskiiı's main lemma ([Sh, Chapter 3, §5, Lemma 8]).

We remark that it is possible to obtain weaker estimations about some of our results, however Proposition 2.4 brings us sharper results. See [N2].

Proposition 2.4 (Shidlovskiu-Osgood's inequality $[\mathrm{O}]$ ). Let $D$ be a simply connected domain in $\mathbb{C}$, and suppose that $D$ does not contain singular points of $A$ in (EQ). Assume that a vector solution $m:=\left(f_{1}, \ldots, f_{n}\right)$ of (EQ) is analytic on $D$, and $f_{1}, \ldots, f_{n}$ are linearly independent over $\mathbb{C}(x)$. Then there exists a constant $c<\infty$, independent of $N$ and $D$, such that

$$
\sum_{t \in D} \max \left(\operatorname{ord}_{x=t} \sum_{i=1}^{n} P_{i} f_{i}-(n-1), 0\right) \leq n N+c
$$

for any $N \in \mathbb{N}$ and any $P_{1}, \ldots, P_{n} \in K[x]$ with $\max _{i=1, \ldots, n} \operatorname{deg} P_{i}<N$. In particular, if $V$ is a finite set $\subset D$, then there exists a constant $c<\infty$ satisfying

$$
\sum_{\zeta \in V}\left(\operatorname{ord}_{x=\zeta} \sum_{i=1}^{n} P_{i} f_{i}\right) \leq n N+(n-1) \# V+c .
$$

See Theorem IV in $[\mathrm{O}]$ for the details. 
The following is the so-called Siegel lemma, which is due to Bombieri [B].

Proposition 2.5 (Siegel's lemma [B]). Let $D_{K}$ be the discriminant of $K$, and $\gamma:=4 d_{K}^{2 d_{K}}\left|D_{K}\right|^{1 / 2}$. Let $M, N \in \mathbb{N}$ with $M<N$ and $a_{i, j} \in K$ for $i=1, \ldots, M, j=1, \ldots, N$. Then there exists a non-trivial solution $\bar{x}=\left(x_{1}, \ldots, x_{N}\right) \in K^{N} \backslash\{\overline{0}\}$ of the system

$$
\sum_{j=1}^{N} a_{i, j} x_{j}=0 \quad \text { for } i=1, \ldots, M
$$

which satisfies

$$
h(\bar{x}) \leq \frac{1}{N-M} \sum_{i=1}^{M} \sum_{v \in M_{K}} \max _{j=1, \ldots, N} \log \left|a_{i, j}\right|_{v}+\frac{M}{N-M} \log (2 N \gamma)+\log \gamma .
$$

See $[\mathrm{B}]$ for the proof.

3. An inequality. The aim of this section is to show a fundamental inequality (Lemma 3.4 below) which will be used in the proofs of our results. The idea of the proof is to consider Padé approximations of $m$ in (EQ) using Siegel's lemma, and combine them with two product formulas, the product formula in a number field and Jensen's formula.

According to $\S 2$, we may say the following: Padé approximations and Jensen's formula are function-theoretic (i.e., geometric), while Siegel's lemma and the product formula in a number field are arithmetic. We recall $\S 2$ : "a geometric property holds together with an arithmetic property". Therefore they can be combined into an inequality. That is Lemma 3.4.

We need long calculations in this section, but each calculation is simple.

3.1. Padé approximations. In this subsection, we will consider Padé approximations at several points and estimate coefficients of the Padé polynomials by Siegel's lemma.

Let $\phi$ be a column vector of infinitely differentiable functions: $\phi \in\left(\mathcal{C}^{\infty}\right)^{n}$. For $A$ in (EQ), we put

$$
\begin{aligned}
& \left(\frac{d}{d x}+{ }^{\mathrm{t}} A\right)^{0} \phi:=\phi, \quad\left(\frac{d}{d x}+{ }^{\mathrm{t}} A\right)^{1} \phi:=\frac{d}{d x} \phi+{ }^{\mathrm{t}} A \phi \\
& \left(\frac{d}{d x}+{ }^{\mathrm{t}} A\right)^{s} \phi:=\left(\frac{d}{d x}+{ }^{\mathrm{t}} A\right)\left(\frac{d}{d x}+{ }^{\mathrm{t}} A\right)^{s-1} \phi \quad \text { for } s=1,2, \ldots
\end{aligned}
$$

LEMMA 3.1. Let $m$ be a vector solution of (EQ), and suppose that $\phi \in\left(\mathcal{C}^{\infty}\right)^{n}$. Then for $s=0,1, \ldots$ we have

$$
\left(\frac{d}{d x}\right)^{s}\left({ }^{\mathrm{t}} \phi m\right)={ }^{\mathrm{t}}\left(\left(\frac{d}{d x}+{ }^{\mathrm{t}} A\right)^{s} \phi\right) m \text {. }
$$


Proof. Since $(d / d x) m=A m$, we obtain (3.1) from

$$
\frac{d}{d x}\left({ }^{\mathrm{t}} \phi m\right)=\left(\frac{d}{d x}{ }^{\mathrm{t}} \phi\right) m+{ }^{\mathrm{t}} \phi A m={ }^{\mathrm{t}}\left(\left(\frac{d}{d x}+{ }^{\mathrm{t}} A\right) \phi\right) m .
$$

Lemma 3.2. Suppose that $\phi \in\left(\mathcal{C}^{\infty}\right)^{n}$. Then for $s=0,1, \ldots$ we have

$$
\frac{1}{s !}\left(\frac{d}{d x}+{ }^{\mathrm{t}} A\right)^{s} \phi=\sum_{\substack{\mu+\nu=s \\ \mu, \nu \geq 0}} \frac{1}{\mu ! \nu !}\left(\left(\frac{d}{d x}+{ }^{\mathrm{t}} A\right)^{\mu} I\right)\left(\left(\frac{d}{d x}\right)^{\nu} \phi\right) .
$$

Here $I$ is the identity matrix.

Proof. For $s=0$, the left side of (3.2) is $\phi$, and the right side is $I \phi=\phi$. For $s=1$, the left side of $(3.2)$ is $(d / d x) \phi+{ }^{\mathrm{t}} A \phi$, and the right side is $I(d / d x) \phi+{ }^{\mathrm{t}} A I \phi$. Thus we have (3.2) for $s=0$ and $s=1$.

We use induction on $s$. Assume that (3.2) holds for a given $s$. Then

$$
\begin{aligned}
\left(\frac{d}{d x}+{ }^{\mathrm{t}} A\right)^{s+1} \phi= & \left(\frac{d}{d x}+{ }^{\mathrm{t}} A\right)\left(\frac{d}{d x}+{ }^{\mathrm{t}} A\right)^{s} \phi \\
= & \left(\frac{d}{d x}+{ }^{\mathrm{t}} A\right) \sum_{\mu+\nu=s} \frac{s !}{\mu ! \nu !}\left(\left(\frac{d}{d x}+{ }^{\mathrm{t}} A\right)^{\mu} I\right)\left(\left(\frac{d}{d x}\right)^{\nu} \phi\right) \\
= & \sum_{\mu+\nu=s} \frac{s !}{\mu ! \nu !}\left(\frac{d}{d x}\left(\frac{d}{d x}+{ }^{\mathrm{t}} A\right)^{\mu} I\right)\left(\left(\frac{d}{d x}\right)^{\nu} \phi\right) \\
& +\frac{s !}{\mu ! \nu !}\left(\left(\frac{d}{d x}+{ }^{\mathrm{t}} A\right)^{\mu} I\right)\left(\frac{d}{d x}\right)^{\nu+1} \phi \\
& +\frac{s !}{\mu ! \nu !}{ }^{\mathrm{t}} A\left(\left(\frac{d}{d x}+{ }^{\mathrm{t}} A\right)^{\mu} I\right)\left(\frac{d}{d x}\right)^{\nu} \phi \\
= & \sum_{\mu+\nu=s} \frac{s !}{\mu ! \nu !}\left(\left(\frac{d}{d x}+{ }^{\mathrm{t}} A\right)^{\mu+1} I\right)\left(\left(\frac{d}{d x}\right)^{\nu} \phi\right) \\
& +\frac{s !}{\mu ! \nu !}\left(\left(\frac{d}{d x}+{ }^{\mathrm{t}} A\right)^{\mu} I\right)\left(\left(\frac{d}{d x}\right)^{\nu+1} \phi\right) \\
= & \sum_{\mu+\nu=s+1} \frac{(s+1) !}{\mu ! \nu !}\left(\left(\frac{d}{d x}+{ }^{\mathrm{t} A}\right)^{\mu} I\right)\left(\left(\frac{d}{d x}\right)^{\nu} \phi\right) .
\end{aligned}
$$

Here we used the following simple formula: if $\mathcal{A}$ is a $\mathbb{Q}$-algebra and $\left\{a_{i}\right\}_{i},\left\{b_{j}\right\}_{j} \subset \mathcal{A}$, then by induction

$$
\sum_{\substack{i+j=k \\ i, j \geq 0}} \frac{k !}{i ! j !}\left(a_{i+1} b_{j}+a_{i} b_{j+1}\right)=\sum_{\substack{i+j=k+1 \\ i, j \geq 0}} \frac{(k+1) !}{i ! j !} a_{i} b_{j} .
$$


Now, let $N$ be a parameter in $\mathbb{N}$, take $P_{1}, \ldots, P_{n} \in K[x]$ with $\max _{i} \operatorname{deg} P_{i}$ $<N$ as Padé polynomials and put

$$
\phi:={ }^{\mathrm{t}}\left(P_{1}, \ldots, P_{n}\right)={ }^{\mathrm{t}}\left(\sum_{j=0}^{N-1} a_{1, j} x^{j}, \ldots, \sum_{j=0}^{N-1} a_{n, j} x^{j}\right) .
$$

We also let $\alpha_{\zeta} \in \mathbb{Z}_{\geq 0}$ be parameters, and we assume that

$$
\operatorname{ord}_{x=\zeta}{ }^{\mathrm{t}} \phi m \geq \alpha_{\zeta},
$$

that is, for $s=0,1, \ldots, \alpha_{\zeta}-1$,

$$
\left(\frac{1}{s !}\left(\frac{d}{d x}\right)^{s}\left({ }^{\mathrm{t}} \phi m\right)\right)_{\mid x=\zeta}=0 .
$$

Here, $m$ is a vector solution in (EQ), $D$ is as in Theorem A, and we suppose that $\zeta$ in $D \cap K$ satisfies the following: there exist $\kappa_{\zeta} \in \mathbb{C} \backslash\{0\}$ and $\theta_{1}, \ldots, \theta_{n} \in K$ such that

$$
m_{\mid x=\zeta}=\kappa_{\zeta}{ }^{\mathrm{t}}\left(\theta_{1}, \ldots, \theta_{n}\right) .
$$

Moreover we assume that $\zeta$ is not a pole of

$$
\left(\frac{d}{d x}+{ }^{\mathrm{t}} A\right)^{\mu} I
$$

for $\mu=0,1, \ldots$ The last assumption holds if $d(\zeta) \neq 0$ by Lemma 2.1.

Now, since $m$ is a solution of (EQ), by Lemma 3.1, the condition

$$
\left(\frac{1}{s !}\left(\frac{d}{d x}\right)^{s}\left({ }^{\mathrm{t}} \phi m\right)\right)_{\mid x=\zeta}=0
$$

is equivalent to

$$
\left(\frac{1}{s !}^{\mathrm{t}}\left(\left(\frac{d}{d x}+{ }^{\mathrm{t}} A\right)^{s} \phi\right) m\right)_{\mid x=\zeta}=0
$$

and also to

$$
\sum_{\substack{\mu+\nu=s \\ \mu, \nu \geq 0}}\left({ }^{\mathrm{t}}\left(\frac{1}{\mu !}\left(\left(\frac{d}{d x}+{ }^{\mathrm{t}} A\right)^{\mu} I\right) \frac{1}{\nu !}\left(\frac{d}{d x}\right)^{\nu} \phi\right) m\right)_{\mid x=\zeta}=0
$$

by Lemma 3.2. Clearly, the latter is equivalent to

$$
\sum_{\substack{\mu+\nu=s \\ \mu, \nu \geq 0}}\left({ }^{\mathrm{t}}\left(\frac{1}{\mu !}\left(\left(\frac{d}{d x}+{ }^{\mathrm{t}} A\right)^{\mu} I\right) \frac{1}{\nu !}\left(\frac{d}{d x}\right)^{\nu} \phi\right) \kappa_{\zeta}^{-1} m\right)_{\mid x=\zeta}=0 .
$$

We put

$$
\left(\alpha_{i, j}(\mu)\right)_{i, j=1, \ldots, n}:=\left(\frac{1}{\mu !}\left(\frac{d}{d x}+{ }^{\mathrm{t}} A\right)^{\mu} I\right)_{\mid x=\zeta} \in M_{n}(K) .
$$


Since $\zeta$ is not a pole, we have

$$
\begin{aligned}
\left(\left(\frac{1}{\mu !}\left(\frac{d}{d x}+{ }^{\mathrm{t}} A\right)^{\mu} I\right)\right. & \left.\left(\frac{1}{\nu !}\left(\frac{d}{d x}\right)^{\nu} \phi\right)\right)_{\mid x=\zeta} \\
& =\left(\frac{1}{\mu !}\left(\frac{d}{d x}+{ }^{\mathrm{t}} A\right)^{\mu} I\right)_{\mid x=\zeta}\left(\frac{1}{\nu !}\left(\frac{d}{d x}\right)^{\nu} \phi\right)_{\mid x=\zeta},
\end{aligned}
$$

that is,

$$
\begin{aligned}
\left(\alpha_{i, j}(\mu)\right)_{i, j=1, \ldots, n}\left(\begin{array}{c}
\sum_{j=0}^{N-1}\left(\begin{array}{c}
j \\
\nu
\end{array}\right) \zeta^{j-\nu} a_{1, j} \\
\vdots \\
\sum_{j=0}^{N-1}\left(\begin{array}{c}
j \\
\nu
\end{array}\right) \zeta^{j-\nu} a_{n, j}
\end{array}\right) & \\
& =\left(\begin{array}{c}
\sum_{j=0}^{N-1} \sum_{k=1}^{n} \alpha_{1, k}(\mu)\left(\begin{array}{l}
j \\
\nu
\end{array}\right) \zeta^{j-\nu} a_{k, j} \\
\vdots \\
\sum_{j=0}^{N-1} \sum_{k=1}^{n} \alpha_{n, k}(\mu)\left(\begin{array}{l}
j \\
\nu
\end{array}\right) \zeta^{j-\nu} a_{k, j}
\end{array}\right) .
\end{aligned}
$$

Hence

$$
\begin{aligned}
{ }^{\mathrm{t}}\left({ } ^ { \mathrm { t } } \left(\left(\frac{1}{\mu !}\left(\frac{d}{d x}+{ }^{\mathrm{t}} A\right)^{\mu} I\right)_{\mid x=\zeta}(\right.\right. & \left.\left.\left(\frac{1}{\nu !}\left(\frac{d}{d x}\right)^{\nu} \phi\right)_{\mid x=\zeta}\right)\left(\kappa_{\zeta}^{-1}\right) m_{\mid x=\zeta}\right) \\
& =\sum_{i=1}^{n} \sum_{k=1}^{n} \sum_{j=0}^{N-1} \theta_{i} \alpha_{i, k}(\mu)\left(\begin{array}{l}
j \\
\nu
\end{array}\right) \zeta^{j-\nu} a_{k, j} .
\end{aligned}
$$

Therefore

$$
\begin{aligned}
\sum_{\substack{\mu+\nu=s \\
\mu, \nu \geq 0}}\left({ }^{\mathrm{t}}\left(\frac{1}{\mu !}\left(\left(\frac{d}{d x}+{ }^{\mathrm{t}} A\right)^{\mu} I\right) \frac{1}{\nu !}\left(\frac{d}{d x}\right)^{\nu} \phi\right) \kappa_{\zeta}^{-1} m\right)_{\mid x=\zeta} \\
=\sum_{k=1}^{n} \sum_{j=0}^{N-1}\left(\sum_{i=1}^{n} \sum_{\substack{\mu+\nu=s \\
\mu, \nu \geq 0}} \theta_{i} \alpha_{i, k}(\mu)\left(\begin{array}{l}
j \\
\nu
\end{array}\right) \zeta^{j-\nu}\right) a_{k, j},
\end{aligned}
$$

where $\left(\begin{array}{l}j \\ \nu\end{array}\right)=0$ if $j<\nu$.

We regard (3.3) as linear combinations $a_{k, j}$. Each coefficient of $a_{k, j}$ is

$$
\sum_{i=1}^{n} \sum_{\substack{\mu+\nu=s \\
\mu, \nu \geq 0}} \theta_{i} \alpha_{i, k}(\mu)\left(\begin{array}{l}
j \\
\nu
\end{array}\right) \zeta^{j-\nu} .
$$

We will find an upper bound of (3.4) for each $k=1, \ldots, n$ and $j=0, \ldots$ $\ldots, N-1$.

We consider two cases. 
Case $v \nmid \infty$ : We have

$$
|(3.4)|_{v} \leq \max _{\substack{i, k=1, \ldots, n \\
j=0, \ldots, N-1 \\
\mu+\nu=s \\
\mu, \nu \geq 0}}\left|\theta_{i}\right|_{v}\left|\alpha_{i, k}(\mu)\right|_{v}\left|\left(\begin{array}{l}
j \\
\nu
\end{array}\right)\right|_{v}\left|\zeta^{j-\nu}\right|_{v} .
$$

Since $\left|\left(\begin{array}{l}j \\ \mu\end{array}\right)\right|_{v} \leq 1$, we obtain

$$
\prod_{v \nmid \infty}|(3.4)|_{v} \leq \prod_{v \nmid \infty} \max _{i=1, \ldots, n}\left|\theta_{i}\right|_{v} \prod_{v \nmid \infty} \max _{\substack{\mu \leq s \\ i, j=1, \ldots, n}}\left|\alpha_{i, j}(\mu)\right|_{v} \prod_{v \nmid \infty} \max \left(1,|\zeta|_{v}\right)^{N-1} .
$$

Case $v \mid \infty$ : We have

$$
|(3.4)|_{v} \leq \max _{\substack{i, k=1, \ldots, n \\
j=0, \ldots, N-1 \\
\mu+\nu=s \\
\mu, \nu \geq 0}}|n(s+1)|_{v}\left|\theta_{i}\right|_{v}\left|\alpha_{i, k}(\mu)\right|_{v}\left|\left(\begin{array}{l}
j \\
\nu
\end{array}\right)\right|_{v}\left|\zeta^{j-\nu}\right|_{v} .
$$

As $\left|\left(\begin{array}{l}j \\ \mu\end{array}\right)\right|_{v} \leq\left|2^{j}\right|_{v}$, we obtain

$\prod_{v \mid \infty}|(3.4)|_{v}$

$\leq 2^{N-1} n(s+1) \prod_{v \nmid \infty} \max _{i=1, \ldots, n}\left|\theta_{i}\right|_{v} \prod_{v \nmid \infty} \max _{\substack{\mu \leq s \\ i, j=1, \ldots, n}}\left|\alpha_{i, j}(\mu)\right|_{v} \prod_{v \nmid \infty} \max \left(1,|\zeta|_{v}\right)^{N-1}$.

In (2.5), we established that

$$
\prod_{v \nmid \infty}\left|\frac{d^{\mu}}{\mu !}\left(\frac{d}{d x}+{ }^{\mathrm{t}} A\right)^{\mu} I\right|_{v} \leq G_{0}(\mu), \quad \prod_{v \mid \infty}\left|\frac{d^{\mu}}{\mu !}\left(\frac{d}{d x}+{ }^{\mathrm{t}} A\right)^{\mu} I\right|_{v} \leq G_{\infty}(\mu),
$$

and by Lemma 2.1,

$$
\operatorname{deg} \frac{d^{\mu}}{\mu !}\left(\frac{d}{d x}+{ }^{\mathrm{t}} A\right)^{\mu} I \leq \mu \gamma_{1}
$$

Since $d(\zeta) \neq 0$, for $v \nmid \infty$ we have

$$
\begin{aligned}
\mid\left(\frac{1}{\mu !}\left(\frac{d}{d x}+{ }^{\mathrm{t}} A\right)^{\mu}\right. & I)\left._{\mid x=\zeta}\right|_{v} \\
& \leq\left|\frac{d^{\mu}}{\mu !}\left(\frac{d}{d x}+{ }^{\mathrm{t}} A\right)^{\mu} I\right|_{v} \max \left(1,|\zeta|_{v}\right)^{\mu \gamma_{1}} \max \left(1,|1 / d(\zeta)|_{v}\right)^{\mu} .
\end{aligned}
$$

Therefore

$$
\begin{aligned}
\prod_{v \nmid \infty} \max _{\mu \leq s}\left|\alpha_{i, j}(\mu)\right|_{v} & =\prod_{v \nmid \infty} \max _{\mu \leq s}\left|\left(\frac{1}{\mu !}\left(\frac{d}{d x}+{ }^{\mathrm{t}} A\right)^{\mu} I\right)_{x=\zeta}\right|_{v} \\
& \leq G_{0}(s) \prod_{v \nmid \infty} \max \left(1,|\zeta|_{v}^{s \gamma_{1}}\right) \prod_{v \nmid \infty} \max \left(1,|1 / d(\zeta)|_{v}^{s}\right) .
\end{aligned}
$$


For $v \mid \infty$, we have

$$
\begin{aligned}
& \left|\left(\frac{1}{\mu !}\left(\frac{d}{d x}+{ }^{\mathrm{t}} A\right)^{\mu} I\right)_{\mid x=\zeta}\right|_{v} \\
& \quad \leq\left|\mu \gamma_{1}+1\right|_{v}\left|\frac{d^{\mu}}{\mu !}\left(\frac{d}{d x}+{ }^{\mathrm{t}} A\right)^{\mu} I\right|_{v} \max \left(1,|\zeta|_{v}\right)^{\mu \gamma_{1}} \max \left(1,|1 / d(\zeta)|_{v}\right)^{\mu} .
\end{aligned}
$$

Therefore

$$
\begin{aligned}
\prod_{v \mid \infty} \max _{\mu \leq s}\left|\alpha_{i, j}(\mu)\right|_{v} & =\prod_{v \mid \infty} \max _{\mu \leq s}\left|\left(\frac{1}{\mu !}\left(\frac{d}{d x}+{ }^{\mathrm{t}} A\right)^{\mu} I\right)_{\mid x=\zeta}\right|_{v} \\
& \leq\left(s \gamma_{1}+1\right) G_{\infty}(s) \prod_{v \mid \infty} \max \left(1,|\zeta|_{v}^{s \gamma_{1}}\right) \prod_{v \mid \infty} \max \left(1,|1 / d(\zeta)|_{v}^{s}\right) .
\end{aligned}
$$

To summarize the above calculations, we arrive at the following: Let $D$ be as in Theorem A, let $\zeta \in K \cap D$ with $d(\zeta) \neq 0$, and $\alpha_{\zeta} \in \mathbb{Z}_{\geq 0}$. Suppose that $m$ is a vector solution of (EQ), analytic on $D$.

Moreover suppose that there exist $\kappa_{\zeta} \in \mathbb{C} \backslash\{0\}$ and $\theta_{1}, \ldots, \theta_{n} \in K$ such that

$$
m_{\mid x=\zeta}=\kappa_{\zeta}{ }^{\mathrm{t}}\left(\theta_{1}, \ldots, \theta_{n}\right)
$$

We consider

$$
\phi=\left(\sum_{j=0}^{\mathrm{t}} a_{1, j} x^{j}, \ldots, \sum_{j=0}^{N-1} a_{n, j} x^{j}\right)
$$

with parameters $a_{k, j}, k=1, \ldots, n, j=0, \ldots, N-1$. Then the system

$$
\left(\frac{1}{s !}\left(\frac{d}{d x}\right)^{s}\left({ }^{\mathrm{t}} \phi m\right)\right)_{\mid x=\zeta}=0, \quad s=0,1, \ldots, \alpha_{\zeta}-1
$$

multiplied by $\kappa_{\zeta}^{-1}$ is equivalent to

$$
\text { the right side of }(3.3)=0, \quad s=0,1, \ldots, \alpha_{\zeta}-1 \text {. }
$$

These are linear equations in $a_{k, j}$ over $K$. The coefficient of $a_{k, j}$ equals (3.4) and satisfies

$$
\begin{aligned}
\prod_{v \nmid \infty}|(3.4)|_{v} \leq & G_{0}\left(\alpha_{\zeta}-1\right) \prod_{v \nmid \infty} \max _{i}\left|\theta_{i}\right|_{v} \prod_{v \nmid \infty} \max \left(1,|\zeta|_{v}\right)^{N-1+\alpha_{\zeta} \gamma_{1}} \\
& \times \prod_{v \nmid \infty} \max \left(1,|1 / d(\zeta)|_{v}\right)^{\alpha_{\zeta}-1},
\end{aligned}
$$

and 


$$
\begin{aligned}
\prod_{v \mid \infty}|(3.4)|_{v} \leq & \left(\left(\alpha_{\zeta}-1\right) \gamma_{1}+1\right) 2^{N-1} n \alpha_{\zeta} G_{\infty}\left(\alpha_{\zeta}-1\right) \prod_{v \mid \infty} \max _{i}\left|\theta_{i}\right|_{v} \\
& \times \prod_{v \mid \infty} \max \left(1,|\zeta|_{v}\right)^{N-1+\alpha_{\zeta} \gamma_{1}} \prod_{v \mid \infty} \max \left(1,|1 / d(\zeta)|_{v}\right)^{\alpha_{\zeta}-1}
\end{aligned}
$$

We note that $\theta_{1}, \ldots, \theta_{n}$ are independent of $N, \alpha_{\zeta}$, and $G_{0}, G_{\infty}, \gamma_{1}$ are independent of $\zeta$.

To combine them into one inequality, we have

$$
\begin{aligned}
\prod_{v \in M_{K}}|(3.4)|_{v} & \\
\leq & \left(\left(\alpha_{\zeta}-1\right) \gamma_{1}+1\right) 2^{N-1} n \alpha_{\zeta} G_{0}\left(\alpha_{\zeta}-1\right) G_{\infty}\left(\alpha_{\zeta}-1\right) \\
& \times\left(\prod_{v \in M_{K}} \max _{i}\left|\theta_{i}\right|_{v}\right) H(\zeta)^{N-1+\left(\alpha_{\zeta}-1\right) \gamma_{1}} H(1 / d(\zeta))^{\alpha_{\zeta}-1}
\end{aligned}
$$

We will apply the above argument to Siegel's lemma.

Let $\zeta_{0}, \ldots, \zeta_{l}$ be $l+1$ distinct elements in $D \cap K$ with $d\left(\zeta_{t}\right) \neq 0$ such that $m$ is analytic at $x=\zeta_{t}$ for $t=0, \ldots, l$. Moreover for $t=0, \ldots, l$, we assume that there exist $\kappa_{t} \in \mathbb{C} \backslash\{0\}$ and $\theta_{1, t}, \ldots, \theta_{n, t} \in K$ such that

$$
m_{\mid x=\zeta_{t}}=\kappa_{t}{ }^{\mathrm{t}}\left(\theta_{1, t}, \ldots, \theta_{n, t}\right) .
$$

Now let $\phi$ be as above, and let $\alpha_{0}, \ldots, \alpha_{l}$ in $\mathbb{Z}_{\geq 0}$ be given. We consider the condition

$$
\operatorname{ord}_{x=\zeta_{t}}\left({ }^{\mathrm{t}} \phi m\right) \geq \alpha_{t} \quad \text { for } t=0, \ldots, l .
$$

These inequalities are equivalent to the homogeneous linear equations over $K$ :

$$
\left(\kappa_{t}^{-1}\right)\left(\frac{1}{s !}\left(\frac{d}{d x}\right)^{s}\left({ }^{\mathrm{t}} \phi m\right)\right)_{\mid x=\zeta_{t}}=0 \quad \text { for } s=0, \ldots, \alpha_{t}-1, t=0, \ldots, l .
$$

Here, the number of equations is $\sum_{t=0}^{l} \alpha_{t}$, and the number of unknowns (i.e., the coefficients of $x^{i}$ in $\phi$ for $i=0, \ldots, N-1$ ) is $n N$.

Now recall Siegel's lemma of $\S 2$.

From (3.5), the value corresponding to $\sum_{i=1}^{M} \sum_{v \in M_{K}} \max _{j} \log \left|a_{i, j}\right|_{v}$ in Siegel's lemma is

$$
\begin{aligned}
& \sum_{t=0}^{l} \alpha_{t}\left(\log G_{0}\left(\alpha_{t}-1\right)+\log G_{\infty}\left(\alpha_{t}-1\right)+\sum_{v \in M_{K}} \log \max _{i}\left|\theta_{i, t}\right|_{v}\right. \\
& \quad+\left(N-1+\left(\alpha_{t}-1\right) \gamma_{1}\right) \sum_{v \in M_{K}} \log ^{+}\left|\zeta_{t}\right|_{v}+\left(\alpha_{t}-1\right) \sum_{v \in M_{K}} \log ^{+}\left|1 / d\left(\zeta_{t}\right)\right|_{v} \\
& \left.\quad+(N-1) \log 2+\log \left(\left(\alpha_{t}-1\right) \gamma_{1}+1\right)+\log n+\log \alpha_{t}\right) .
\end{aligned}
$$


Since $h(a)=h(1 / a)$ for $a \in K \backslash\{0\}$, applying (3.6) in Siegel's lemma, we obtain:

LEMmA 3.3. Let $l$ be a given non-negative integer, and let $\zeta_{0}, \ldots, \zeta_{l}$ be $l+1$ distinct elements in $D \cap K$ with $d\left(\zeta_{0}\right) \neq 0, \ldots, d\left(\zeta_{l}\right) \neq 0$. Suppose that there exist $\kappa_{t} \in \mathbb{C} \backslash\{0\}$ and $\theta_{1, t}, \ldots, \theta_{n, t} \in K$ such that

$$
m_{\mid x=\zeta_{t}}=\kappa_{t}{ }^{\mathrm{t}}\left(\theta_{1, t}, \ldots, \theta_{n, t}\right) \quad \text { for } t=0, \ldots, l .
$$

Let $\alpha_{0}, \ldots, \alpha_{l} \in \mathbb{Z}_{\geq 0}$, and let $\delta$ be a positive number with

$$
(n-\delta) N=\sum_{t=0}^{l} \alpha_{t}
$$

Then for any $N \in \mathbb{N}$, there exists a non-trivial $\phi \in(K[x])^{n} \backslash\{\overline{0}\}$ with $\operatorname{deg} \phi<N$ such that

$$
\operatorname{ord}_{x=\zeta_{t}}\left({ }^{\mathrm{t}} \phi m\right) \geq \alpha_{t} \quad \text { for } t=0, \ldots, l
$$

and

$$
\begin{aligned}
h(\phi) \leq & \frac{1}{\delta N} \sum_{t=0}^{l} \alpha_{t}\left(\log G_{0}\left(\alpha_{t}-1\right)+\log G_{\infty}\left(\alpha_{t}-1\right)+(N-1) \log 2\right. \\
& +\left(N-1+\left(\alpha_{t}-1\right) \gamma_{1}\right) h\left(\zeta_{t}\right)+\left(\alpha_{t}-1\right) h\left(d\left(\zeta_{t}\right)\right) \\
& \left.+\sum_{v \in M_{K}} \log \max _{i}\left|\theta_{i, t}\right|_{v}+\log \left(\left(\left(\alpha_{t}-1\right) \gamma_{1}+1\right) n \alpha_{t}\right)\right) \\
& +\frac{n-\delta}{\delta} \log (2 n N \gamma)+\log \gamma .
\end{aligned}
$$

Here $\gamma$ is defined in Siegel's lemma.

3.2. Jensen's formula. In this subsection, we recall the classical Jensen formula ([L2, p. 162]). We will then combine it with Lemma 3.3 in the last subsection.

Let $R>0$ and $\varepsilon>0$ be given. For $\zeta \in \mathbb{C}$, we put $\Delta(\zeta, R):=\{z \in \mathbb{C} \mid$ $|z-\zeta| \leq R\}$. Let $g$ be a meromorphic function on $\Delta(0, R+\varepsilon)$. Then Jensen's formula reads:

$-\sum_{\substack{a \in \Delta(0, R) \\ a \neq 0}} \operatorname{ord}_{x=a}(g) \log \frac{R}{|a|}-\lambda \log R-\log \left|c_{\lambda}\right|+\frac{1}{2 \pi} \int_{0}^{2 \pi} \log \left|g\left(R e^{\sqrt{-1} \theta}\right)\right| d \theta=0$ for $g(z)=c_{\lambda} z^{\lambda}+c_{\lambda+1} z^{\lambda+1}+\ldots, c_{\lambda} \neq 0, \lambda \in \mathbb{Z}$.

Now let $\left\{\alpha_{i}\right\}_{i}$ be the set of zeros and poles of $g(z)$ on $\Delta(0, R)$. Put $f(z):=g(z-\zeta)$. Then $f$ is a meromorphic function on $\Delta(\zeta, R+\varepsilon)$ with $f(z)=c_{\lambda}(z-\zeta)^{\lambda}+c_{\lambda+1}(z-\zeta)^{\lambda+1}+\ldots, c_{\lambda} \neq 0$, and $\left\{\alpha_{i}+\zeta\right\}_{i}$ is the set of zeros and poles of $f(z)$ on $\Delta(\zeta, R)$. 
Putting $\beta_{i}:=\alpha_{i}+\zeta$, we have by Jensen's formula

$$
\begin{aligned}
-\sum_{\beta_{i} \neq \zeta} \operatorname{ord}_{z=\beta_{i}}(f(z)) \log \frac{R}{\left|\beta_{i}-\zeta\right|} & -\lambda \log R-\log \left|c_{\lambda}\right| \\
& +\frac{1}{2 \pi} \int_{0}^{2 \pi} \log \left|f\left(R e^{\sqrt{-1} \theta}+\zeta\right)\right| d \theta=0 .
\end{aligned}
$$

We note that $\log \left(R /\left|\beta_{i}-\zeta\right|\right) \geq 0$ because $R \geq\left|\alpha_{i}\right|=\left|\beta_{i}-\zeta\right|$.

If $f(z)$ is analytic (i.e., has no poles) on $\Delta(\zeta, R+\varepsilon)$, then for any subset $Z \subset\left\{\beta_{i}\right\}$ we have

$$
\begin{aligned}
-\sum_{\substack{a \in Z \\
a \neq \zeta}}\left(\log _{z=a} f(z)\right) \log \frac{R}{|a-\zeta|} & -\lambda \log R-\log \left|c_{\lambda}\right| \\
+ & \frac{1}{2 \pi} \int_{0}^{2 \pi} \log \left|f\left(R e^{\sqrt{-1} \theta}+\zeta\right)\right| d \theta \geq 0,
\end{aligned}
$$

where $f(z)=c_{\lambda}(z-\zeta)^{\lambda}+c_{\lambda+1}(z-\zeta)^{\lambda+1}+\ldots, \lambda \geq 0, c_{\lambda} \neq 0$.

Now we consider ${ }^{\mathrm{t}} \phi m$ for $\phi$ as in Lemma 3.3. We suppose that $\zeta_{0}$ belongs to $D$. We choose $R$ satisfying $\Delta\left(\zeta_{0}, R\right) \subset D$ and assume that $\zeta_{0}, \ldots, \zeta_{l} \in$ $\Delta\left(\zeta_{0}, R\right) \cap K$. We put

$$
\beta_{t}:=\operatorname{ord}_{x=\zeta_{t}}\left({ }^{\mathrm{t}} \phi m\right) \quad \text { for } t=0, \ldots, l .
$$

Obviously, $\beta_{t} \geq \alpha_{t}$ for $t=0, \ldots, l$.

If $\beta_{t}=\infty$, then ${ }^{\mathrm{t}} \phi m$ is 0 on a neighborhood of $\zeta_{t}$, and thus ${ }^{\mathrm{t}} \phi m$ is 0 on $D$ by the uniqueness theorem. This $m$ does not satisfy the assumptions of Theorem A.

Set $\zeta:=\zeta_{0}$ and put

$$
\psi_{0}:=\kappa_{0}^{-1}\left(\frac{1}{x-\zeta}\right)^{\beta_{0}} .
$$

Since $\operatorname{ord}_{x=\zeta}\left(\psi_{0}{ }^{\mathrm{t}} \phi m\right)=0$ and $\psi_{0}{ }^{\mathrm{t}} \phi m \neq 0$, by (3.8) we have $-\sum_{t=1}^{l} \beta_{t} \log \frac{R}{\left|\zeta_{t}-\zeta\right|}-\log \left|\psi_{0}{ }^{\mathrm{t}} \phi m_{\mid x=\zeta}\right|+\frac{1}{2 \pi} \int_{0}^{2 \pi} \log \left|\psi_{0}{ }^{\mathrm{t}} \phi m_{\mid x=R e^{\sqrt{-1} \theta}+\zeta}\right| d \theta \geq 0$, where we assume that $R$ satisfies $\Delta(\zeta, R) \subset D$.

Now, we will find an upper bound of

$$
\frac{1}{2 \pi} \int_{0}^{2 \pi} \log \left|\psi_{0}^{\mathrm{t}} \phi m_{\mid x=R e^{\sqrt{-1} \theta}+\zeta}\right| d \theta
$$

Since

$$
\left|\psi_{0}{ }^{\mathrm{t}} \phi m\right|=\left|\kappa_{0}^{-1}\left(\frac{1}{x-\zeta}\right)^{\beta_{0}}{ }^{\mathrm{t}} \phi m\right|
$$


we have

$$
\begin{aligned}
\frac{1}{2 \pi} \int_{0}^{2 \pi} \log \left|\psi_{0}{ }^{\mathrm{t}} \phi m_{\mid x=R e^{\sqrt{-1} \theta}+\zeta}\right| d \theta & \\
& =\frac{1}{2 \pi} \int_{0}^{2 \pi} \log \left|\kappa_{0}^{-1 \mathrm{t}} \phi m_{\mid x=R e^{\sqrt{-1} \theta}+\zeta}\right| d \theta-\beta_{0} \log R .
\end{aligned}
$$

From now on, let $T_{v} \in \mathbb{R}\left(v \in M_{K}\right)$ be such that

$$
|\phi|_{v} \leq T_{v} \text {. }
$$

Using the usual absolute valuations $|\ldots|$, for $v=\mathrm{id}=1$ we obtain

$$
|\phi|=|\phi|_{1}^{d_{K} / d_{1}} \leq T_{1}^{d_{K} / d_{1}} .
$$

Hence

$$
\max _{i, j}\left|a_{i, j}\right| \leq T_{1}^{d_{K} / d_{1}} \quad \text { for } \quad \phi={ }^{\mathrm{t}}\left(\sum_{j=0}^{N-1} a_{1, j} x^{j}, \ldots, \sum_{j=0}^{N-1} a_{n, j} x^{j}\right) .
$$

We put $m={ }^{\mathrm{t}}\left(f_{1}(x), \ldots, f_{n}(x)\right)$ in $D$. Since

$$
{ }^{\mathrm{t}} \phi m=\sum_{i=1}^{n} \sum_{j=0}^{N-1} a_{i, j} x^{j} f_{i}(x)
$$

we have

$$
\left|\kappa_{0}^{-1 \mathrm{t}} \phi m_{\mid x=R e^{\sqrt{-1} \theta}+\zeta}\right| \leq \sum_{i=1}^{n} \sum_{j=0}^{N-1}\left|a_{i, j}\right|(R+|\zeta|)^{j}\left|\kappa_{0}^{-1}\right| \max _{\substack{i=1, \ldots, n \\ 0 \leq \theta \leq 2 \pi}}\left|f_{i}\left(R e^{\sqrt{-1} \theta}+\zeta\right)\right| .
$$

Now set

$$
M(R, \zeta):=\left|\kappa_{0}^{-1}\right| \max _{\substack{i=1, \ldots, n \\ 0 \leq \theta \leq 2 \pi}}\left|f_{i}\left(R e^{\sqrt{-1} \theta}+\zeta\right)\right| .
$$

Then

$$
\begin{aligned}
& \frac{1}{2 \pi} \int_{0}^{2 \pi} \log \left|\kappa_{0}^{-1 \mathrm{t}} \phi m_{\mid x=R e^{\sqrt{-1} \theta}+\zeta}\right| d \theta \\
& \quad \leq \frac{d_{K}}{d_{1}} \log T_{1}+\log \max \left(1,(R+|\zeta|)^{N-1}\right)+\log n N+\log M(R, \zeta) .
\end{aligned}
$$

We note that $M(R, \zeta)$ is independent of $N$.

By (3.8), we conclude that

$$
\begin{aligned}
& -\beta_{0} \log R-\sum_{t=1}^{l} \beta_{t} \log \frac{R}{\left|\zeta_{t}-\zeta\right|}-\log \left|\psi_{0}{ }^{\mathrm{t}} \phi m_{\mid x=\zeta}\right| \\
& +\frac{d_{K}}{d_{1}} \log T_{1}+\log \max \left(1,(R+|\zeta|)^{N-1}\right)+\log n N+\log M(R, \zeta) \geq 0 .
\end{aligned}
$$


3.3. Product formula in the number field. In this subsection, we will find upper bounds of

$$
\sum_{v \in M_{K}^{0}} \log \left|\psi_{0}{ }^{\mathrm{t}} \phi m_{\mid x=\zeta}\right|_{v} \quad \text { and } \quad \sum_{\sigma \in M_{K}^{1}} \frac{d_{\sigma}}{d_{K}} \log \left|\sigma\left(\psi_{0}{ }^{\mathrm{t}} \phi m_{\mid x=\zeta}\right)\right| .
$$

Here $\sigma(a)$ is the image of $a \in K$ under $\sigma$.

Now, since $\beta_{0}=\operatorname{ord}_{x=\zeta}\left({ }^{\mathrm{t}} \phi m\right)$, we have

$$
\left(\frac{\kappa_{0}^{-1}}{\beta_{0} !}\left(\frac{d}{d x}\right)^{\beta_{0}}\left({ }^{\mathrm{t}} \phi m\right)\right)_{\mid x=\zeta}=\left(\psi_{0}{ }^{\mathrm{t}} \phi m\right)_{\mid x=\zeta} .
$$

From Lemmas 3.1 and 3.2, we have

$$
\frac{\kappa_{0}^{-1}}{\beta_{0} !}\left(\frac{d}{d x}\right)^{\beta_{0}}\left({ }^{\mathrm{t}} \phi m\right)=\left(\sum_{\substack{\mu+\nu=\beta_{0} \\ \mu, \nu \geq 0}}\left(\frac{1}{\mu !}\left(\frac{d}{d x}+{ }^{\mathrm{t}} A\right)^{\mu} I\right)\left(\frac{1}{\nu !}\left(\frac{d}{d x}\right)^{\nu} \phi\right)\right) \kappa_{0}^{-1} m .
$$

We recall that $|\phi|_{v} \leq T_{v}$ and $\operatorname{deg} \phi<N$.

We note that

$$
\frac{1}{\nu !}\left(\frac{d}{d x}\right)^{\nu} \phi={ }^{\mathrm{t}}\left(\sum_{j=0}^{N-1}\left(\begin{array}{l}
j \\
\nu
\end{array}\right) a_{1, j} x^{j}, \ldots, \sum_{j=0}^{N-1}\left(\begin{array}{l}
j \\
\nu
\end{array}\right) a_{n, j} x^{j}\right) .
$$

From Lemma 2.1, let $A_{j}(\mu) \in M_{n}(K)$ be such that

$$
A(\mu):=\frac{d^{\mu}}{\mu !}\left(\frac{d}{d x}+{ }^{\mathrm{t}} A\right)^{\mu} I=\sum_{j=0}^{\mu \gamma_{1}} A_{j}(\mu) x^{j} \in M_{n}(K[x]) .
$$

Since $d(\zeta) \neq 0$, we have

$$
\left(\frac{1}{\mu !}\left(\frac{d}{d x}+{ }^{\mathrm{t}} A\right)^{\mu} I\right)_{\mid x=\zeta}=\frac{A(\mu)_{\mid x=\zeta}}{d(\zeta)^{\mu}}
$$

and

$$
\begin{aligned}
& \left(\frac{\kappa_{0}^{-1}}{\beta_{0} !}\left(\frac{d}{d x}\right)^{\beta_{0}}\left({ }^{\mathrm{t}} \phi m\right)\right)_{\mid x=\zeta} \\
= & \sum_{\substack{\mu+\nu=\beta_{0} \\
\mu, \nu \geq 0}}\left(\left(\frac{1}{\mu !}\left(\frac{d}{d x}+{ }^{\mathrm{t}} A\right)^{\mu} I\right)_{\mid x=\zeta}\left(\frac{1}{\nu !}\left(\frac{d}{d x}\right)^{\nu} \phi\right)_{\mid x=\zeta}\right) \kappa_{0}^{-1} m_{\mid x=\zeta} .
\end{aligned}
$$

We consider two cases. 
Case $v \nmid \infty$ : We have

$$
\begin{aligned}
\mid\left(\psi_{0}{ }^{\mathrm{t}} \phi m\right)_{|x=\zeta|_{v}=} & \left|\left(\frac{\kappa_{0}^{-1}}{\beta_{0} !}\left(\frac{d}{d x}\right)^{\beta_{0}}\left({ }^{\mathrm{t}} \phi m\right)\right)_{\mid x=\zeta}\right|_{v} \\
\leq & \max _{\mu=0, \ldots, \beta_{0}}\left|\left(\frac{d^{\mu}}{\mu !}\left(\frac{d}{d x}+{ }^{\mathrm{t}} A\right)^{\mu} I\right)_{\mid x=\zeta}\right|_{v} \max _{\mu=0, \ldots, \beta_{0}}\left|\frac{1}{d(\zeta)^{\mu}}\right|_{v} \\
& \times \max _{\nu=0, \ldots, \beta_{0}}\left|\left(\frac{1}{\nu !}\left(\frac{d}{d x}\right)^{\nu} \phi\right)_{\mid x=\zeta}\right|_{v}\left|\kappa_{0}^{-1} m_{\mid x=\zeta}\right|_{v} .
\end{aligned}
$$

Here $\left|\kappa_{0}^{-1} m_{\mid x=\zeta}\right|_{v}:=\max _{i}\left|\theta_{i, 0}\right|_{v}$. From Lemma 2.1, since

$$
\operatorname{deg} \frac{d^{\mu}}{\mu !}\left(\frac{d}{d x}+{ }^{\mathrm{t}} A\right) I \leq \beta_{0} \gamma_{1}, \quad \operatorname{deg} \frac{1}{\nu !}\left(\frac{d}{d x}\right)^{v} \phi \leq N-1
$$

for $\mu, \nu \leq \beta_{0}$, we have

$$
\begin{aligned}
\prod_{v \nmid \infty} \mid\left(\psi_{0}^{\mathrm{t}} \phi m\right)_{|x=\zeta|_{v} \leq} & G_{0}\left(\beta_{0}\right) \prod_{v \nmid \infty} \max \left(1,\left|\frac{1}{d(\zeta)}\right|_{v}^{\beta_{0}}\right) \prod_{v \nmid \infty} T_{v} \prod_{v \nmid \infty}\left|\kappa_{0}^{-1} m_{\mid x=\zeta}\right|_{v} \\
& \times \prod_{v \nmid \infty} \max \left(1,|\zeta|_{v}\right)^{N-1+\beta_{0} \gamma_{1}} .
\end{aligned}
$$

We rewrite the last inequality as

$$
\begin{aligned}
\sum_{v \nmid \infty} \log \left|\left(\psi_{0}{ }^{\mathrm{t}} \phi m\right)_{\mid x=\zeta}\right|_{v} \leq & \log G_{0}\left(\beta_{0}\right)+\beta_{0} \sum_{v \nmid \infty} \log ^{+}\left|\frac{1}{d(\zeta)}\right|_{v} \\
& +\sum_{v \nmid \infty} \log T_{v}+\sum_{v \nmid \infty} \log \left|\kappa_{0}^{-1} m_{\mid x=\zeta}\right|_{v} \\
& +\left(N-1+\beta_{0} \gamma_{1}\right) \sum_{v \nmid \infty} \log ^{+}|\zeta|_{v} .
\end{aligned}
$$

Case $v \mid \infty$ : Note that

$$
\left(\frac{d^{\mu}}{\mu !}\left(\frac{d}{d x}+{ }^{\mathrm{t}} A\right)^{\mu} I\right)_{\mid x=\zeta}, \quad\left(\frac{1}{\nu !}\left(\frac{d}{d x}\right)^{\nu} \phi\right)_{\mid x=\zeta}, \quad \kappa_{0}^{-1} m_{\mid x=\zeta}
$$

are $n \times n-, n \times 1-, 1 \times n$-matrices respectively, with entries in $K$. By (3.11),

$$
\begin{gathered}
\left|\left(\psi_{0}{ }^{\mathrm{t} \phi m}\right)_{\mid x=\zeta}\right|_{v}=\left|\left(\frac{\kappa_{0}^{-1}}{\beta_{0} !}\left(\frac{d}{d x}\right)^{\beta_{0}}\left({ }^{\mathrm{t}} \phi m\right)\right)_{\mid x=\zeta}\right|_{v} \\
\leq\left|\beta_{0}+1\right|_{v}\left|n^{2}\right|_{v}\left(\max _{\mu \leq \beta_{0}}\left|\frac{1}{d(\zeta)^{\mu}}\right|_{v}\right)\left(\max _{\mu \leq \beta_{0}}\left|\left(\frac{d^{\mu}}{\mu !}\left(\frac{d}{d x}+{ }^{\mathrm{t} A}\right)^{\mu} I\right)_{\mid x=\zeta}\right|_{v}\right) \\
\times\left(\max _{\nu \leq \beta_{0}}\left|\left(\frac{1}{\nu !}\left(\frac{d}{d x}\right)^{\nu} \phi\right)_{\mid x=\zeta}\right|_{v}\right)\left|\kappa_{0}^{-1} m_{\mid x=\zeta}\right|_{v} .
\end{gathered}
$$


By (3.12), we obtain

$$
\begin{aligned}
& \sum_{v \in M_{K}^{1}} \log \mid\left(\psi_{0}{ }^{\mathrm{t}} \phi m\right)_{|x=\zeta|} \\
& \leq \beta_{0} \sum_{v \in M_{K}^{1}} \log ^{+}|1 / d(\zeta)|_{v}+\log G_{1}\left(\beta_{0}\right)+\sum_{v \in M_{K}^{1}} \log T_{v} \\
& \quad+\sum_{v \in M_{K}^{1}} \log \left|\kappa_{0}^{-1} m_{\mid x=\zeta}\right|_{v}+\sum_{v \in M_{K}^{1}} \log \left|\left(\beta_{0} \gamma_{1}+1\right) N\left(\beta_{0}+1\right) n^{2}\right|_{v} \\
& \quad+\left(N-1+\beta_{0} \gamma_{1}\right) \sum_{v \in M_{K}^{1}} \log ^{+}|\zeta|_{v}+(N-1) \log 2
\end{aligned}
$$

by $(3.10)$ since $\left(\begin{array}{l}j \\ \nu\end{array}\right) \leq 2^{N-1}$.

Since $\left(\psi_{0}{ }^{\mathrm{t}} \phi m\right)_{\mid x=\zeta} \in K \backslash\{0\}$, the product formula reads

$$
\begin{aligned}
\log \left|\left(\psi_{0}{ }^{\mathrm{t}} \phi m\right)_{\mid x=\zeta}\right|_{1} & +\sum_{v \in M_{K}^{1}} \log \mid \\
& \left.+\psi_{0}{ }^{\mathrm{t} \phi m}\right)_{|x=\zeta|_{v}} \log \left|\left(\psi_{0}{ }^{\mathrm{t}} \phi m\right)_{\mid x=\zeta}\right|_{v}=0 . \\
& +M_{K}^{0}
\end{aligned}
$$

In the next subsection, we will use the last equation together with the estimates obtained in this subsection.

3.4. A fundamental inequality. Multiplying (3.9) by $d_{1} / d_{K}$, and combining it with (3.13)-(3.15), we obtain

$$
\begin{aligned}
\beta_{0} \log R^{d_{1} / d_{K}}+\sum_{t=1}^{l} \beta_{t} \log \frac{R^{d_{1} / d_{K}}}{\left|\zeta_{t}-\zeta\right|_{1}} \\
\leq \sum_{v \in M_{K}} \log T_{v}+\frac{d_{1}}{d_{K}}\left(\log ^{+}(R+|\zeta|)^{N-1}+\log n N+\log M(R, \zeta)\right) \\
\quad+\log G_{0}\left(\beta_{0}\right)+\log G_{1}\left(\beta_{0}\right)+\beta_{0} \sum_{\substack{v \in M_{K} \\
v \neq \text { id }}} \log ^{+}\left|\frac{1}{d(\zeta)}\right|_{v} \\
\quad+\sum_{\substack{v \in M_{K} \\
v \neq \mathrm{id}}} \log \left|\kappa_{0}^{-1} m_{\mid x=\zeta}\right|_{v}+\left(N-1+\beta_{0} \gamma_{1}\right) \sum_{\substack{v \in M_{K} \\
v \neq \mathrm{id}}} \log ^{+}|\zeta|_{v} \\
\quad+(N-1) \log 2+\sum_{\substack{v \in M_{K}^{1}\\
}} \log \left|\left(\beta_{0} \gamma_{1}+1\right) N\left(\beta_{0}+1\right) n^{2}\right|_{v} .
\end{aligned}
$$

Applying Lemma 3.3 to the last inequality, since the $T_{v}$ can be supposed to satisfy $\sum_{v \in M_{K}} \log T_{v}=h(\phi)$ and by (3.7), we obtain the long inequality: 


$$
\begin{aligned}
& \beta_{0} \log R^{d_{1} / d_{K}}+\sum_{t=1}^{l} \beta_{t} \log \frac{R^{d_{1} / d_{K}}}{\left|\zeta_{t}-\zeta\right|_{1}} \\
& \leq \frac{1}{\delta N} \sum_{t=0}^{l} \alpha_{t}\left(\log G_{0}\left(\alpha_{t}-1\right)+\log G_{\infty}\left(\alpha_{t}-1\right)+(N-1) \log 2\right. \\
& +\left(N-1+\left(\alpha_{t}-1\right) \gamma_{1}\right) h\left(\zeta_{t}\right)+\left(\alpha_{t}-1\right) h\left(d\left(\zeta_{t}\right)\right) \\
& \left.+\sum_{v \in M_{K}} \log \max _{i}\left|\theta_{i, t}\right|_{v}+\log \left(\left(\left(\alpha_{t}-1\right) \gamma_{1}+1\right) n \alpha_{t}\right)\right) \\
& +\frac{n-\delta}{\delta} \log (2 n N \gamma)+\log \gamma \\
& +\frac{d_{1}}{d_{K}}\left(\log ^{+}\left((R+|\zeta|)^{N-1}\right)+\log (n N)+\log M(R, \zeta)\right) \\
& +\log G_{0}\left(\beta_{0}\right)+\log G_{1}\left(\beta_{0}\right)+\beta_{0} \sum_{\substack{v \in M_{K} \\
v \neq 1}} \log ^{+}|1 / d(\zeta)|_{v} \\
& +\sum_{\substack{v \in M_{K} \\
v \neq 1}} \log \left|\kappa_{0}^{-1} m_{\mid x=\zeta}\right|_{v}+\left(N-1+\beta_{0} \gamma_{1}\right) \sum_{\substack{v \in M_{K} \\
v \neq 1}} \log ^{+}|\zeta|_{v} \\
& +(N-1) \log 2+\sum_{v \in M_{K}^{1}} \log \left|\left(\beta_{0} \gamma_{1}+1\right) N\left(\beta_{0}+1\right) n^{2}\right|_{v} .
\end{aligned}
$$

We remark that (3.16) holds in the following sense: for any $N=1,2, \ldots$ and any $\alpha_{0}, \ldots, \alpha_{l}$ with $\sum_{t=0}^{l} \alpha_{t}=(n-\delta) N$, there exist $\beta_{0}, \ldots, \beta_{l} \in \mathbb{Z}_{\geq 0}$ such that

$$
\beta_{t} \geq \alpha_{t} \quad \text { for } t=0, \ldots, l, \quad \sum_{t=0}^{l} \beta_{t} \leq n N+c(l+1)
$$

and (3.16) holds.

Here the inequality $\sum_{t=0}^{l} \beta_{t} \leq n N+c(l+1)$ comes from ShidlovskiuOsgood's inequality of $\S 2$ and $c$ is a finite constant depending only on $m$.

Now, we assume that $(\mathrm{EQ})$ is a $G$-operator, and thus we assume that there exists a constant $C<\infty$, depending only on $A$, such that $G_{0}(\mu) \leq C^{\mu}$ for $\mu=0,1, \ldots$

From $\S 2$, for a given $a \geq 0$ and $N \in \mathbb{N}$, there exist $C_{0}, C_{\infty}, C_{1}$ such that

$$
\frac{\log G_{0}(a N)}{N} \leq a C_{0}, \quad \frac{\log G_{\infty}(a N)}{N} \leq a C_{\infty}, \quad \frac{\log G_{1}(a N)}{N} \leq a C_{1} .
$$

Let $a_{0}, \ldots, a_{l} \in \mathbb{R}_{\geq 0}$ and $b_{0}, \ldots, b_{l} \in \mathbb{R}_{\geq 0}$ satisfy

$$
\alpha_{t}=a_{t} N, \quad \beta_{t}=b_{t} N
$$


We assume that $R+|\zeta|<1$. We note that $\theta_{i, t}$ and $M(R, \zeta)$ are independent of $N$.

Dividing the long inequality (3.16) by $N$, and letting $N \rightarrow \infty$, from $R+|\zeta|<1$ we have

$$
\begin{aligned}
\sum_{t=0}^{l} b_{t} \log R^{d_{1} / d_{k}}+\sum_{t=1}^{l} b_{t} \log \frac{1}{\left|\zeta_{t}-\zeta\right|_{1}} \\
\leq \frac{1}{\delta} \sum_{t=0}^{l} a_{t}\left(\left(C_{0}+C_{\infty}\right) a_{t}+\log 2+\left(1+a_{t} \gamma_{1}\right) h\left(\zeta_{t}\right)+a_{t} h\left(d\left(\zeta_{t}\right)\right)+\varepsilon\right) \\
\quad+2 \varepsilon+\left(C_{0}+C_{1}\right) b_{0}+b_{0} h\left(d\left(\zeta_{0}\right)\right)+\varepsilon+\left(1+b_{0} \gamma_{1}\right) h\left(\zeta_{0}\right)+\log 2+\varepsilon
\end{aligned}
$$

for any positive $\varepsilon>0$.

Since $\operatorname{deg} d \leq \gamma_{1}$, there exists $\gamma_{2}$ such that $h(d(\zeta)) \leq \gamma_{1} h(\zeta)+\gamma_{2}$ for any $\zeta \in K$ (e.g., see [Se, p. 15]).

Consequently, we arrive at:

Lemma 3.4 (The fundamental inequality). Let $D$ be as in Theorem A and let $m$ be an analytic vector solution of (EQ) satisfying the assumptions in Theorem A. Assume that $\zeta_{0}, \ldots, \zeta_{l} \in D \cap K$ are such that there exists $\kappa_{t} \in \mathbb{C} \backslash\{0\}$ with $\kappa_{t} m\left(\zeta_{t}\right) \in K^{n}$. Assume that $d\left(\zeta_{t}\right) \neq 0, t=0, \ldots, l$. Let $R$ be a positive number with $R+\left|\zeta_{0}\right|<1$ and assume that $\zeta_{1}, \ldots, \zeta_{l} \in$ $\Delta\left(\zeta_{0}, R\right) \subset D$. Then under the assumptions of Theorem $\mathrm{A}$, for any $\delta>0$ with $\delta<n$ and for any $a_{0}, \ldots, a_{t} \in \mathbb{R}_{\geq 0}$ with $\sum_{t=0}^{l} a_{t}=n-\delta$, there exist $b_{0}, \ldots, b_{l} \in \mathbb{R}_{\geq 0}$ with $b_{t} \geq a_{t}$ for $t=0, \ldots, l$ satisfying the following: For any positive $\varepsilon>0$,

$$
\sum_{t=0}^{l} b_{t} \leq n+\varepsilon
$$

and

$$
\begin{aligned}
& \sum_{t=0}^{l} b_{t} \log R^{d_{1} / d_{K}}+\sum_{t=1}^{l} b_{t} \log \frac{1}{\left|\zeta_{t}-\zeta_{0}\right|_{1}} \\
& \leq \frac{1}{\delta} \sum_{t=0}^{l} a_{t}\left(\left(1+2 a_{t} \gamma_{1}\right) h\left(\zeta_{t}\right)+a_{t}\left(C_{0}+C_{\infty}+\gamma_{2}\right)+\log 2+\varepsilon\right) \\
& \quad+\left(1+2 b_{0} \gamma_{1}\right) h\left(\zeta_{0}\right)+b_{0}\left(C_{0}+C_{1}+\gamma_{2}\right)+\log 2+\varepsilon
\end{aligned}
$$

Here $\gamma_{1}, \gamma_{2}, C_{0}, C_{1}, C_{\infty}$ are finite constants depending only on $A$.

Remark. The condition $R+|\zeta|<1$ is not essential for two reasons. One can assume without loss of generality that $|\zeta|<1 / 2$, because the definition of $G$-operators is independent of the choice of coordinates. Moreover large $R$ 's make only slight changes in (3.17). 


\section{Proofs}

4.1. Proof of Theorem A. First, we will show an ineffective version, and next we prove the effectiveness.

We use Lemma 3.4. Put $l:=1$, fix $\zeta_{0}$ and put $a_{0}:=0$. Dividing (3.17) by $h\left(\zeta_{1}\right)$, we have

$$
\begin{aligned}
\frac{\left(b_{0}+b_{1}\right) \log R^{d_{1} / d_{K}}}{h\left(\zeta_{1}\right)}+b_{1} \frac{\log \left(1 /\left|\zeta_{0}-\zeta_{1}\right|_{1}\right)}{h\left(\zeta_{1}\right)} \\
\leq \frac{1}{\delta} a_{1}\left(\left(1+2 a_{1} \gamma_{1}\right)+\frac{a_{1}\left(C_{0}+C_{\infty}+\gamma_{2}\right)+\log 2+\varepsilon}{h\left(\zeta_{1}\right)}\right) \\
\quad+\frac{\left(1+2 b_{0} \gamma_{1}\right) h\left(\zeta_{0}\right)+b_{0}\left(C_{0}+C_{1}+\gamma_{2}\right)+\log 2+\varepsilon}{h\left(\zeta_{1}\right)} .
\end{aligned}
$$

If $h\left(\zeta_{1}\right)$ is much larger than $n, h\left(\zeta_{0}\right), R, C_{0}, C_{1}, C_{\infty}, \gamma_{1}$ and $\gamma_{2}$, we obtain

$$
-\varepsilon+b_{1} \frac{\log \left(1 /\left|\zeta_{0}-\zeta_{1}\right|_{v}\right)}{h\left(\zeta_{1}\right)} \leq \frac{a_{1}}{\delta}\left(\left(1+2 a_{1} \gamma_{1}\right)+\varepsilon\right)+\varepsilon .
$$

We can assume that $\log \left(1 /\left|\zeta_{1}-\zeta_{0}\right|_{1}\right)>0$ because if $\left|\zeta_{1}-\zeta_{0}\right|_{1} \geq 1$ we need to do nothing. Since $b_{1} \geq a_{1}$, (4.1) implies that

$$
a_{1} \frac{\log \left(1 /\left|\zeta_{1}-\zeta_{0}\right|_{1}\right)}{h\left(\zeta_{1}\right)} \leq \frac{a_{1}}{\delta}\left(\left(1+2 a_{1} \gamma_{1}\right)+\varepsilon\right)+2 \varepsilon .
$$

We assume that $a_{1}>0$ and divide the last inequality by $a_{1}$ to obtain

$$
\frac{\log \left(1 /\left|\zeta_{1}-\zeta_{0}\right|_{1}\right)}{h\left(\zeta_{1}\right)} \leq \frac{1}{\delta}\left(\left(1+2 a_{1} \gamma_{1}\right)+\varepsilon\right)+\frac{2 \varepsilon}{a_{1}} .
$$

Now, let $\varepsilon_{1}>0$. We put $a_{1}:=\varepsilon_{1}$, and thus $\varepsilon_{1}=a_{1}=a_{0}+a_{1}=n-\delta$. We can assume that $\varepsilon \leq \varepsilon_{1}^{2}$; consequently,

$$
\frac{\log \left(1 /\left|\zeta_{1}-\zeta_{0}\right|_{1}\right)}{h\left(\zeta_{1}\right)} \leq \frac{1}{n-\varepsilon_{1}}\left(1+\varepsilon_{1}\left(2 \gamma_{1}+\varepsilon_{1}\right)\right)+2 \varepsilon_{1} .
$$

Therefore, for any given small $\varepsilon_{2}>0$, if $h\left(\zeta_{1}\right)$ is large, we have

$$
\frac{\log \left(1 /\left|\zeta_{1}-\zeta_{0}\right|_{1}\right)}{h\left(\zeta_{1}\right)} \leq \frac{1}{n}+\varepsilon_{2}
$$

that is,

$$
\frac{1}{\left|\zeta_{1}-\zeta_{0}\right|_{1}} \leq H\left(\zeta_{1}\right)^{1 / n+\varepsilon_{2}}
$$

Because we just need to consider the case of $\left|\zeta_{1}-\zeta_{0}\right|<1$, we can assume that $\left|\zeta_{1}-\zeta_{0}\right|_{1}=\left|\zeta_{1}-\zeta_{0}\right|^{d_{1} / d_{K}} \leq\left|\zeta_{1}-\zeta_{0}\right|^{1 / d_{K}}$. Therefore we conclude that the ineffective version of Theorem A holds. 
Now we show the effectiveness. From Lemma 3.4, we can assume that $b_{0}+b_{1} \leq n+\varepsilon_{1}$. Here we can assume that $\varepsilon=\varepsilon_{1}^{2}$. Since $\gamma_{1} \geq 1$, the inequality

$$
\frac{1}{n-\varepsilon_{1}}\left(1+\varepsilon_{1}\left(2 \gamma_{1}+1\right)\right)+2 \varepsilon_{1} \leq \frac{1}{n}+\varepsilon_{2}
$$

holds if we put $\varepsilon_{1}\left(2 \gamma_{1}+1\right)=\varepsilon_{2} / 4$ for small $\varepsilon_{1}$.

We have to find a sufficient condition (which involves $\varepsilon_{2}$ ) for the following three inequalities to hold:

$$
\begin{gathered}
-\varepsilon \leq \frac{\left(b_{0}+b_{1}\right) \log R^{d_{1} / d_{K}}}{h\left(\zeta_{1}\right)}, \quad \frac{a_{1}\left(C_{0}+C_{\infty}+\gamma_{2}\right)+\log 2+\varepsilon}{h\left(\zeta_{1}\right)} \leq \varepsilon, \\
\frac{\left(1+2 b_{0} \gamma_{1}\right) h\left(\zeta_{0}\right)+b_{0}\left(C_{0}+C_{1}+\gamma_{2}\right)+\log 2+\varepsilon}{h\left(\zeta_{1}\right)} \leq \varepsilon .
\end{gathered}
$$

It is easy to verify that there exist $c_{1}, c_{2}>0$ such that these three inequalities hold if

$$
h\left(\zeta_{1}\right) \geq \frac{c_{1} h\left(\zeta_{0}\right)+c_{2}}{\varepsilon_{2}^{2}},
$$

where $c_{1}$ and $c_{2}$ are obtained from $n, R, C_{0}, C_{1}, C_{\infty}, \gamma_{1}$ and $\gamma_{2}$, that is, they are effective constants depending only on $A$ and $R$.

4.2. Proof of Theorem B. One can say that Theorem A is a Liouville inequality for $G$-functions on fixed targets. In this subsection, we consider a variant of Liouville's inequality on moving targets. Here we use Lemma 3.4 as well.

We consider only the cases where $\zeta_{0}, \ldots, \zeta_{l}$ are close to each other in the topology of $|\ldots|_{1}$. We replace the index 0 of $\zeta_{0}$ in Lemma 3.4 with another index $i$, that is, we consider Lemma 3.4 with $\zeta_{i}$ (resp. $\left.\Delta\left(\zeta_{i}, R\right)\right)$ in place of $\zeta_{0}\left(\right.$ resp. $\left.\Delta\left(\zeta_{0}, R\right)\right)$. Under the notations of Lemma 3.4, we have

$$
\begin{aligned}
\sum_{t=0}^{l} b_{t} \log R^{d_{1} / d_{K}}+\sum_{\substack{t=0 \\
t \neq i}}^{l} b_{t} \log \frac{1}{\left|\zeta_{t}-\zeta_{i}\right|_{1}} \\
\leq \frac{1}{\delta} \sum_{t=0}^{l} a_{t}\left(\left(1+2 a_{t} \gamma_{1}\right) h\left(\zeta_{t}\right)+a_{t}\left(C_{0}+C_{\infty}+\gamma_{2}\right)+\log 2+\varepsilon\right) \\
+\left(1+2 b_{i} \gamma_{1}\right) h\left(\zeta_{i}\right)+b_{i}\left(C_{0}+C_{1}+\gamma_{2}\right)+\log 2+\varepsilon .
\end{aligned}
$$

Now let $Q$ be a large real number and assume that $h\left(\zeta_{t}\right) \leq Q$ for $t=$ $0, \ldots, l$. We also suppose that $\left|\zeta_{t}-\zeta_{i}\right|_{1} \leq 1$. Since $0 \leq a_{t} \leq b_{t}$ for $t=0, \ldots, l$ and since $\sum_{t=0}^{l} b_{t} \leq n+\varepsilon$, the following inequality holds if $Q$ is sufficiently large: 


$$
\begin{aligned}
-\varepsilon+\sum_{\substack{t=0 \\
t \neq i}}^{l} a_{t} & \frac{\log \left(1 /\left|\zeta_{t}-\zeta_{i}\right|_{1}\right)}{Q} \\
& \leq \frac{1}{\delta} \sum_{t=0}^{l} a_{t}\left(\left(1+2 a_{t} \gamma_{1}\right) \frac{h\left(\zeta_{t}\right)}{Q}+\varepsilon\right)+\left(1+2 b_{t} \gamma_{1}\right) \frac{h\left(\zeta_{i}\right)}{Q}+\varepsilon .
\end{aligned}
$$

Now we put $a_{t}:=(n-\delta) /(l+1)$ for $t=0,1, \ldots, l$.

Because $b_{t} \geq 0$ and $\sum_{t=0}^{l} b_{t} \leq n+\varepsilon$, there exists $i_{0}$ such that $b_{i_{0}} \leq$ $(n+\varepsilon) /(l+1)$. Since $h\left(\zeta_{t}\right) / Q \leq 1$, we have

$$
\begin{aligned}
& \frac{n-\delta}{l+1} \sum_{\substack{t=0 \\
t \neq i_{0}}}^{l} \frac{\log \left(1 /\left|\zeta_{t}-\zeta_{i_{0}}\right|_{1}\right)}{Q} \\
& \quad \leq \frac{1}{\delta}\left((n-\delta)(1+\varepsilon)+\frac{2(n-\delta)^{2} \gamma_{1}}{l+1}\right)+\left(1+\frac{2(n+\varepsilon) \gamma_{1}}{l+1}\right)+2 \varepsilon .
\end{aligned}
$$

We put

$$
M:=\min _{\substack{t=0, \ldots, l \\ t \neq i_{0}}} \log \left(1 /\left|\zeta_{t}-\zeta_{i_{0}}\right|_{1}\right)
$$

and $\delta:=n / 2$, and thus we have

$$
\frac{n}{2} \cdot \frac{l}{l+1} \cdot \frac{M}{Q} \leq 2+3 \varepsilon+\frac{(3 n+2 \varepsilon) \gamma_{1}}{l+1} .
$$

Therefore there exists $L$, which depends only on $\varepsilon, n, \gamma_{1}$, such that if $l \geq L$, then

$$
\frac{n}{2} \cdot \frac{M}{Q} \leq 2+4 \varepsilon, \quad \text { that is, } \quad M \leq \frac{4+8 \varepsilon}{n} Q .
$$

We conclude the following: if $h\left(\zeta_{t}\right) \leq Q$ for $t=0, \ldots, l$, and if

$$
\min _{\substack{t=0, \ldots, l \\ t \neq i_{0}}} \log \left(1 /\left|\zeta_{t}-\zeta_{i_{0}}\right|_{1}\right)=M>\frac{4+8 \varepsilon}{n} Q
$$

then $l<L$. In particular, if

$$
\min _{i_{0}=0, \ldots, l, l} \min _{\substack{t=0, \ldots, l \\ t \neq i_{0}}} \log \left(1 /\left|\zeta_{t}-\zeta_{i_{0}}\right|_{1}\right)>\frac{4+8 \varepsilon}{n} Q
$$

then $l<L$. Therefore if

$$
\max _{\substack{i=0, \ldots, l \\ t=0, \ldots, i \neq t}} \log \left|\zeta_{i}-\zeta_{t}\right|_{1}<-\frac{4+\varepsilon_{1}}{n} Q
$$

then $l<L$. Here $\varepsilon_{1}:=8 \varepsilon$. Consequently, we obtain: 
Theorem E. Suppose that $B$ is an arbitrary large number. Under the assumptions of Theorem $\mathrm{A}$, let $D_{s}$ be an arbitrary closed disk with radius $B^{-4(1+\varepsilon) / n} / 2\left(\right.$ in the topology $\left.|\ldots|_{1}\right)$ with $D_{s} \subset D$. Then there exists $L$ such that the number of $\zeta$ 's which have the following properties is at most $L+1$ :

$$
\zeta \in D_{s} \cap K, H(\zeta) \leq B \text { and there exists } \kappa_{\zeta} \in \mathbb{C} \backslash\{0\}
$$

$$
\text { such that } \kappa_{\zeta} m(\zeta) \in K^{n} \text {. }
$$

Here $L$ depends only on $\varepsilon, n, \gamma_{1}$ (independent of $B$ and the choice of the center of $D_{s}$ ).

Now, we give a proof of Theorem B.

Proof of Theorem B. We have to consider two cases: $|\ldots|_{1}=|\ldots|^{1 /[K: \mathbb{Q}]}$ and $|\ldots|_{1}=|\ldots|^{2 /[K: \mathbb{Q}]}$. We only consider the second case. The first case is similar.

Let $R$ be the radius of $D$, the closed disk $\subset \mathbb{C}$ in Theorem B. It is easy to see that $D$ is covered by $8 R^{2} B^{4(1+\varepsilon)[K: \mathbb{Q}] / n}$ small disks with radius $B^{-4(1+\varepsilon)[K: \mathbb{Q}] /(2 n)} / 2$. In each of the small disks the number of $\zeta$ 's satisfying the conditions of Theorem $\mathrm{E}$ is at most $L+1$. Thus the number of $\zeta$ 's in $D$ satisfying the conditions of Theorem $\mathrm{E}$ is at most $(L+1) 8 R^{2} B^{4(1+\varepsilon)[K: \mathbb{Q}] / n}$. Therefore we obtain

$$
\varlimsup_{B \rightarrow \infty} \frac{\log \#\left\{\zeta \in S_{K} \mid H(\zeta) \leq B\right\}}{\log B} \leq \frac{4(1+\varepsilon)}{n}[K: \mathbb{Q}] .
$$

Since the last estimate holds for any $\varepsilon$, we get the conclusion of Theorem B.

4.3. Proof of Corollaries $C$ and $D$. Let $m={ }^{\mathrm{t}}\left(y_{1}, \ldots, y_{n}\right)$ be a vector solution of (EQ). We denote by $m_{N}$ the vector whose components are all monomials in $y_{1}, \ldots, y_{n}$ with degree $N$. For example, if $m={ }^{\mathrm{t}}\left(y_{1}, y_{2}\right)$, then $m_{1}=m, m_{2}={ }^{\mathrm{t}}\left(y_{1}^{2}, y_{1} y_{2}, y_{2}^{2}\right)$, and so on.

Put $N^{\prime}:=\left(\begin{array}{c}n+N \\ n\end{array}\right)$. From [Sh, Lemma 18, p. 118], there exists an $N^{\prime} \times N^{\prime}-$ matrix $A_{N}$ whose components are linear combinations of components of $A$ in (EQ) over $\mathbb{Z}$, such that $m_{N}$ satisfies

$$
\frac{d}{d x} m_{N}=A_{N} m_{N}
$$

Therefore one can take $d$ in Theorem A to be a common denominator of the components of $A_{N}$.

If there exists $\kappa_{\zeta} \in \mathbb{C} \backslash\{0\}$ such that $\kappa_{\zeta} m(\zeta) \in K^{n}$ then $\kappa_{\zeta}^{N} m_{N}(\zeta) \in$ $K^{N^{\prime}}$, and thus

$\left\{\zeta \in K \mid\right.$ there exists $\kappa_{\zeta} \in \mathbb{C} \backslash\{0\}$ such that $\left.\kappa_{\zeta} m(\zeta) \in K^{n}\right\}$

$\subset\left\{\zeta \in K \mid\right.$ there exists $\kappa \in \mathbb{C} \backslash\{0\}$ such that $\left.\kappa m_{N}(\zeta) \in K^{N^{\prime}}\right\}$. 
Under the assumptions of Corollaries $\mathrm{C}$ and D, the elements of $m_{N}$ are linearly independent over $\mathbb{C}(x)$ and from Proposition $2.3,\left(\mathrm{EQ}_{N}\right)$ is a $G$-operator.

Applying Theorems A and B to $\left(\mathrm{EQ}_{N}\right)$ for large $N$, we obtain Corollaries $\mathrm{C}$ and D.

\section{References}

[A] Y. André, G-functions and Geometry, Max-Planck-Institut, Bonn, 1989.

[BMV] F. Beukers, T. Matala-aho and K. Väänänen, Remarks on the arithmetic properties of the values of hypergeometric functions, Acta Arith. 42 (1983), 281-289.

[B] E. Bombieri, On G-functions, in: Recent Progress in Analytic Number Theory 2, Academic Press, 1981, 1-67.

[C] D. V. Chudnovsky and G. V. Chudnovsky, Applications of Padé approximations to Diophantine inequalities in values of $G$-functions, in: Lecture Notes in Math. 1135, Springer, 1985, 9-51.

[G] A. I. Galochkin, Estimates from below of polynomials in the values of analytic functions of a certain class, Math. USSR-Sb. 24 (1974), 385-407.

[L1] S. Lang, Fundamentals of Diophantine Geometry, Springer, 1983.

[L2] - Introduction to Complex Hyperbolic Spaces, Springer, 1987.

[N1] M. Nagata, A generalization of the sizes of differential equations and its applications to G-function, Ann. Scuola Norm. Sup. Pisa Cl. Sci. (4) 30 (2001), 465-497.

[N2] - An estimation on the number of rational values related to G-functions, preprint RIMS (1231).

[N3] - Transformations on G-functions, preprint RIMS (1197).

[O] C. F. Osgood, Nearly perfect systems and effective generalizations of Shidlovski's theorem, J. Number Theory 13 (1981), 515-540.

[Se] J.-P. Serre, Lectures on the Mordell-Weil Theorem, 3rd ed., Vieweg, 1997.

[Sh] A. B. Shidlovskii, Transcendental Numbers, de Gruyter Stud. Math., de Gruyter, 1989.

[Wo] J. Wolfart, Werte hypergeometrischer Funktionen, Invent. Math. 92 (1988), $187-216$.

Research Institute for Mathematical Sciences

Kyoto University

Oiwake-cho, Kitashirakawa, Sakyo-ku

Kyoto, 606-8502, Japan

E-mail: mnagata@kurims.kyoto-u.ac.jp

Received on 30.7.1999

and in revised form on 15.4.2002 